\title{
Maintaining interface equilibrium conditions in compressible multiphase flows using interface capturing
}

\author{
Shahaboddin Alahyari Beig*, Eric Johnsen \\ Mechanical Engineering Department, University of Michigan, Ann Arbor, MI 48109, USA
}

\begin{abstract}
An accurate treatment of material interfaces in compressible multiphase flows poses important challenges for high-resolution numerical methods. Although high-order interface-capturing schemes have been used to accurately simulate gas/liquid interfaces with the Euler equations, these methods can result in temperature spikes at material discontinuities. While this phenomenon is not problematic for Euler simulations, it gives rise to numerical errors when heat conduction is included. In this work, we identify the source of these errors and propose a methodology to prevent their occurrence for various models used to represent gas/liquid interfaces in compressible flows based on a "single-fluid" formulation, in which interfaces are represented by discontinuities in the material properties. Our focus lies in materials (gases and liquids primarily, but also solids) that can be described by a stiffened equation of state, though our approach is generalizable to other relations. We show that numerical approaches that prevent pressure oscillations at interfaces may generate temperature errors, which affect the energy (and pressure) through the heat conduction term. We demonstrate that the material properties entering the equation of state must be computed according to suitable transport equations in conservative or non-conservative forms; the pressure and temperature must be calculated based on the appropriate properties. To verify the analysis and compute problems with gas/liquid interfaces of relevance, we develop a three-dimensional, high-order accurate, solution-adaptive finite difference framework. In particular, we show that temperatures and pressures may be significantly overestimated in calculations of shock-induced bubble collapse in water if temperature errors are not prevented.
\end{abstract}

Keywords:

Compressible multiphase flows, shock waves and interfaces, stiffened equation of state, interface capturing, solution-adaptive method.

2010 MSC: 76L05, 76T10

\footnotetext{
${ }^{*}$ Corresponding author

Email addresses: alahyari@umich.edu (Shahaboddin Alahyari Beig), ejohnsen@umich.edu (Eric Johnsen)
} 


\section{Introduction}

Compressible multiphase flows are central to a number of engineering applications, including cavitation erosion and high-speed combustion. One of the main challenges in accurately simulating these flows lies in simultaneously representing shock waves, interfaces separating fluids of large density ratios and physical diffusion processes, due to spurious numerical errors commonly generated at interfaces, which may eventually affect the entire flow field. The present work focuses on developing Eulerian approaches to accurately simulate shock waves and gas/liquid interfaces, with viscous and heat diffusion included.

Numerical methods for Eulerian simulations of compressible flows with interfaces typically fall in one of two categories, tracking or capturing. In this article, we focus on the latter because it is relatively simple to implement even for high-order methods and is a logical approach to treat physical diffusion; tracking, which includes front-tracking [1] and level-set [2] methods, will not be discussed further here. Similarly to shock capturing, interfaces between fluids of different composition can be captured by regularizing them over a few grid points, while maintaining the correct jump conditions. By adding one transport equation for mass conservation of one of the fluids, an extension of the Euler equations to multiple fluids/phases is seemingly straightforward, as such an equation can be solved in conservative form with standard shock-capturing techniques. However, such a naive implementation has long been known to give rise to spurious pressure oscillations for isolated interfaces between fluids of different material properties (i.e., properties entering the equation of state) [3, 4]. Furthermore, since material interfaces are linearly degenerate, there is no physical mechanism to steepen interfaces, unlike shock waves. Thus, to prevent interfaces from being overly smeared by numerical diffusion, high-order solution-adaptive [5, 6] or sharpening [7 9] techniques are often used in practice.

In this context, Abgrall [3] was the first to recognize that, for interfaces separating two gases of different specific heats ratios $\gamma$, an additional transport equation solved for a distinct function of $\gamma$ in non-conservative (advection) form prevents such oscillations. Shyue [4] later expanded this idea to solving a transport equation for the mass fraction, again in non-conservative form, and to liquids and solids obeying a stiffened equation of state. Johnsen \& Colonius [10] further extended these approaches to high-order Weighted Essentially Non-Oscillatory [WENO, 11] methods to simulate non-spherical bubble collapse 12], which Coralic \& Colonius [13] further refined. Such high-order finite volume methods can be computationally expensive in multiple dimensions. To address this difficulty, finite difference [for gases only, 5, 14, 15] and discontinuous Galerkin [6] methods have been proposed, in which high-order limiting is applied only at discontinuities. In simulations of the compressible Navier-Stokes equations for two gases with different specific heats ratios, Johnsen \& Ham [16. noticed that an inconsistent treatment of temperature causes similar errors and significant temperature undershoots due to the coupling via the heat diffusion term; they proposed approaches to overcome these problems based on $\gamma$ or mass fraction formulations. Although temperature errors may occur 
35 in Euler simulations, they have no influence on the results since temperature is a derived quantity. However, such temperature errors are problematic when accounting for heat diffusion, reacting flows, phase change and other temperature-dependent phenomena.

Recent developments in interface capturing for compressible multiphase flows originated from the sevenequations two-phase flow model [17], in which balance equations for mass, momentum and energy of each 40 phase, as well as an equation for volume fraction evolution, are solved. The additional volume fraction equation prevents the occurrence of spurious pressure oscillations. For many problems of practical importance, five-equations models [e.g., that in 18] describe the physics accurately, in which pressure and velocity equilibria between the phases are assumed; thus, equations for mass balance of each phase, for total momentum and energy, and for the volume fraction evolution are solved. This latter model and extensions thereof have been used to study a wide range of phenomena [18 26]. With these models, a consistent and efficient high-order extension to accurately include heat diffusion and predict temperature has yet to be proposed.

The objective of the present work is to develop a treatment for temperature in compressible multiphase flows that is physically consistent and efficient, and that does not produce spurious errors in simulations of gas/liquid interfaces and shocks, with viscous and heat diffusion included. Our approach is general in that it applies to $\gamma$ [as in 3], mass fraction [as in 4] and volume fraction [e.g., five-equations, 18] models. Through our analysis, we identify the causes for numerical errors caused by an inconsistent treatment of temperature using high-order shock-capturing schemes and show how these errors can be prevented so that accurate simulations with physical diffusion can be performed. The resulting 3D finite difference scheme is high-order accurate, conservative and prevents pressure and temperature errors. Our contribution advances the current understanding of compressible multiphase flows in that it generalizes the methodology of Johnsen \& Ham [16] for gases, in which temperature errors are prevented, to gas/liquid flows and different capturing approaches and extends the work of Coralic \& Colonius [13] to prevent temperature errors in such flows. The article is organized as follows. In 92 the physical model is presented, followed by the numerical models (93). In 4 we discuss the causes for spurious pressure and temperature errors in the presence of heat diffusion 60 and propose an approach to prevent them. We briefly describe the numerical implementation in $\$ 5$ and verify it with rigorous test problems in 8 . 


\section{Physical model}

\subsection{Equations of motion}

Assuming no mass transfer or surface tension, the compressible Navier-Stokes equations govern the gas/liquid flows of interest:

$$
\begin{aligned}
\frac{\partial \rho}{\partial t}+\frac{\partial}{\partial x_{j}}\left(\rho u_{j}\right) & =0 \\
\frac{\partial \rho u_{i}}{\partial t}+\frac{\partial}{\partial x_{j}}\left(\rho u_{i} u_{j}+p \delta_{i j}\right) & =\frac{\partial \tau_{i j}}{\partial x_{j}}, \\
\frac{\partial E}{\partial t}+\frac{\partial}{\partial x_{j}}\left[u_{j}(E+p)\right] & =\frac{\partial}{\partial x_{j}}\left(u_{i} \tau_{i j}-Q_{j}\right),
\end{aligned}
$$

where $\rho$ is the density, $u_{i}$ the velocity vector, $p$ the pressure, $E=\rho e+\rho u_{i} u_{i} / 2$ the total energy, $e$ the internal energy and $\delta_{i j}$ the identity tensor. The viscous stress tensor $\tau_{i j}$ and heat flux $Q_{j}$ are given by:

$$
\tau_{i j}=\mu\left(\frac{\partial u_{i}}{\partial x_{j}}+\frac{\partial u_{j}}{\partial x_{i}}-\frac{2}{3} \frac{\partial u_{k}}{\partial x_{k}} \delta_{i j}\right)+\mu_{B} \frac{\partial u_{k}}{\partial x_{k}} \delta_{i j}, \quad Q_{j}=-\kappa \frac{\partial T}{\partial x_{j}},
$$

where $\mu$ is the dynamic shear viscosity, $\mu_{B}$ the bulk viscosity and $\kappa$ the heat conductivity.

\subsection{Equation of state}

A relation between pressure, temperature and internal energy valid for gases and liquids is required. Although homogeneous equilibrium and tabular relationships have been used for cavitating flows [27, 28], the stiffened equation of state introduced by Le Métayer et al. [29] is a simple and sufficiently accurate model, which has been validated against experiments for shock propagation in water and certain solids, and has been used to simulate cavitating flows [30, 31]. The relationships between pressure, temperature and internal energy are given by:

$$
\begin{aligned}
\rho(e-q) & =\frac{p}{n-1}+\frac{n B}{n-1}, & \text { (pressure-wise) } \\
& =\rho c T+B, & \text { (temperature-wise) }
\end{aligned}
$$

where $n, B, q$, and $c$ are material properties fit to experimental data. For air and water, the relevant constants take the values shown in Table (10). In the limit of ideal gases, $n=\gamma$ represents the specific heat ratio, $c=c_{v}$ is the specific heat at constant volume, and $B$ and $q$ are zero, such that the ideal gas law is recovered. For multiphase flows, we follow a "single-fluid" formulation, in which the same thermodynamic relationship holds in the entire domain, with material interfaces denoted by changes in the material properties, which are advected by the flow. 


\subsection{Multifluid modeling} \\ 2.3.1. Definitions and basic relations}

The volume fraction $\alpha^{(k)}$ is the volume of fluid $k$ divided by the total mixture volume in a control volume, and the mass fraction $z^{(k)}$ represents the mass of fluid $k$ divided by the mass of the mixture in the control volume, with $\sum_{k} \alpha^{(k)}=1$ and $\sum_{k} z^{(k)}=1$. Accordingly, the mixture density $\rho$ is defined:

$$
\rho=\sum_{k} \rho^{(k)} \alpha^{(k)}=\sum_{k} \rho z^{(k)}
$$

where the superscripts $(k)$ denote phase/fluid $k$. Similarly, the internal energy of the mixture per unit mass $e$ is:

$$
e=\sum_{k} z^{(k)} e^{(k)}, \quad \text { and } \quad \rho e=\sum_{k} \rho^{(k)} \alpha^{(k)} e^{(k)} .
$$

The mass fraction and volume fraction are related by:

$$
z^{(k)}=\rho^{(k)} \alpha^{(k)} / \rho, \quad \text { with } \quad \frac{1}{\rho}=\sum_{k} \frac{z^{(k)}}{\rho^{(k)}} .
$$

For ideal gases, with $\gamma$ equivalent to $n$, the following relationship further holds:

$$
\left(\frac{1}{\gamma-1}\right) \frac{1}{M}=\sum_{k} z^{(k)}\left(\frac{1}{\gamma^{(k)}-1}\right) \frac{1}{M^{(k)}} .
$$

In the above relationships, $z^{(k)}, \alpha^{(k)}, \rho^{(k)}$ and $e^{(k)}$ may vary in space and time, but $\gamma^{(k)}$ and $M^{(k)}$ do not.

\subsubsection{Mixture relations}

Although we focus on gas/liquid interfaces that are initially sharp, capturing regularizes these interfaces over a few grid points, so that mixture regions exist. For the transport coefficients, we use the mixture relations of Perigaud \& Saurel [21]: $\mu=\sum_{k} \alpha^{(k)} \mu^{(k)}$ and $\kappa=\sum_{k} \alpha^{(k)} \kappa^{(k)}$. For the thermodynamic quantities, we seek to express the material properties that enter the equation of state in an appropriate form for mixtures. Substituting the pressure-based relation stiffened equation (3a) into Eq. (5) yields:

$$
\frac{p}{(n-1)}+\frac{n B}{(n-1)}+\rho q=\sum_{k} \alpha^{(k)} \frac{p^{(k)}}{\left(n^{(k)}-1\right)}+\sum_{k} \alpha^{(k)} \frac{n^{(k)} B^{(k)}}{\left(n^{(k)}-1\right)}+\sum_{k} \alpha^{(k)} \rho^{(k)} q^{(k)},
$$

Table 1: Relevant constants for the stiffened equation of state 29].

\begin{tabular}{c|cc}
\hline \hline Parameter & Air & Water \\
\hline$n$ & 1.4 & 2.35 \\
$B$ & 0 & $1 \mathrm{GPa}$ \\
$c$ & $0.718 \mathrm{~kJ} / \mathrm{kgK}$ & $1.816 \mathrm{~kJ} / \mathrm{kgK}$ \\
$q$ & 0 & $-1.167 \mathrm{~kJ} / \mathrm{kg}$ \\
\hline \hline
\end{tabular}


while substituting the temperature-based stiffened equation (3b) into Eq. (5) yields:

$$
\rho c T+B+\rho q=\sum_{k} \alpha^{(k)} \rho^{(k)} c^{(k)} T^{(k)}+\sum_{k} \alpha^{(k)} B^{(k)}+\sum_{k} \alpha^{(k)} \rho^{(k)} q^{(k)} .
$$

Assuming isobaric $\left(p^{(k)}=p\right)$ and isothermal $\left(T^{(k)}=T\right)$ closure between the phases [19], it follows that

$$
\begin{gathered}
\frac{1}{n-1}=\sum_{k} \alpha^{(k)}\left(\frac{1}{n^{(k)}-1}\right), \quad \frac{n B}{n-1}=\sum_{k} \alpha^{(k)}\left(\frac{n^{(k)} B^{(k)}}{n^{(k)}-1}\right), \\
B=\sum_{k} \alpha^{(k)} B^{(k)}, \quad \rho q=\sum_{k} \rho^{(k)} \alpha^{(k)} q^{(k)}, \quad \rho c=\sum_{k} \rho^{(k)} \alpha^{(k)} c^{(k)} .
\end{gathered}
$$

As a result, we can reformulate $\rho e$ in terms of volume fractions:

$$
\rho e= \begin{cases}p \sum_{k} \alpha^{(k)} \frac{1}{\left(n^{(k)}-1\right)}+\sum_{k} \alpha^{(k)} \frac{n^{(k)} B^{(k)}}{\left(n^{(k)}-1\right)}+\sum_{k} \rho^{(k)} \alpha^{(k)} q^{(k)} & \text { (pressure-wise), } \\ T \sum_{k} \rho^{(k)} \alpha^{(k)} c^{(k)}+\sum_{k} \alpha^{(k)} B^{(k)}+\sum_{k} \rho^{(k)} \alpha^{(k)} q^{(k)} & \text { (temperature-wise). }\end{cases}
$$

A similar procedure can be followed using the energy relation based on the mass fraction in Eq. (5). For the pressure-wise case,

$$
\frac{p}{\rho(n-1)}+\frac{n B}{\rho(n-1)}+q=\sum_{k} z^{(k)} \frac{p^{(k)}}{\rho^{(k)}\left(n^{(k)}-1\right)}+\sum_{k} z^{(k)} \frac{n^{(k)} B^{(k)}}{\rho^{(k)}\left(n^{(k)}-1\right)}+\sum_{k} z^{(k)} q^{(k)},
$$

while for the temperature-wise relation:

$$
c T+\frac{B}{\rho}+q=\sum_{k} z^{(k)} c^{(k)} T^{(k)}+\sum_{k} z^{(k)} \frac{B^{(k)}}{\rho^{(k)}}+\sum_{k} z^{(k)} q^{(k)} .
$$

Likewise, Eqs. (12) and (13) can be rearranged as:

$$
\begin{aligned}
\left(\frac{1}{n-1}\right) \frac{1}{\rho} & =\sum_{k} z^{(k)}\left(\frac{1}{n^{(k)}-1}\right) \frac{1}{\rho^{(k)}}, \quad\left(\frac{n B}{n-1}\right) \frac{1}{\rho}=\sum_{k} z^{(k)}\left(\frac{n^{(k)} B^{(k)}}{n^{(k)}-1}\right) \frac{1}{\rho^{(k)}}, \\
\frac{B}{\rho} & =\sum_{k} z^{(k)} \frac{B^{(k)}}{\rho^{(k)}}, \quad q=\sum_{k} z^{(k)} q^{(k)}, \quad c=\sum_{k} z^{(k)} c^{(k)} .
\end{aligned}
$$

Thus, $\rho e$ can be written in terms of mass fractions:

$$
\rho e= \begin{cases}p \sum_{k} \rho z^{(k)} \frac{1}{\rho^{(k)}\left(n^{(k)}-1\right)}+\sum_{k} \rho z^{(k)} \frac{n^{(k)} B^{(k)}}{\rho^{(k)}\left(n^{(k)}-1\right)}+\sum_{k} \rho z^{(k)} q^{(k)} & \text { (pressure-wise), } \\ T \sum_{k} \rho z^{(k)} c^{(k)}+\sum_{k} \rho z^{(k)} \frac{B^{(k)}}{\rho^{(k)}}+\sum_{k} \rho z^{(k)} q^{(k)} & \text { (temperature-wise). }\end{cases}
$$

\subsubsection{Transport equation}

With the present "single-fluid" formulation, changes in composition are denoted by changes in material properties, which depend on the mass and/or volume fraction. From fundamental principles, the mass conservation equation for either of the phases, assuming no mass transfer, is:

$$
\frac{\partial}{\partial t}\left(\rho z^{(k)}\right)+\frac{\partial}{\partial x_{j}}\left(\rho z^{(k)} u_{j}\right)=0
$$


The continuity equation can be used to write this equation in advection form:

$$
\frac{\partial z^{(k)}}{\partial t}+u_{j} \frac{\partial z^{(k)}}{\partial x_{j}}=0, \quad \text { or } \quad \frac{\partial f}{\partial t}+u_{j} \frac{\partial f}{\partial x_{j}}=0,
$$

where $f$ is any function of $z$.

\section{Numerical models}

Eqs. (11), (21), (3) and (16), along with an appropriate relations between the mass fraction and the material properties in the equation of state form a closed system. However, the discretization of this system may result in spurious pressure oscillations for flows with variable $n$ if care is not taken $[3,4$. Three main interface-capturing approaches have been used to prevent such errors, by solving the transport equation in a different form, usually non-conservative:

- $\gamma$-based approach: Here, transport equations for specific functions of the material properties entering the equation of state must be solved:

$$
\frac{\partial}{\partial t}\left(\frac{1}{n-1}\right)+u_{j} \frac{\partial}{\partial x_{j}}\left(\frac{1}{n-1}\right)=0, \quad \frac{\partial}{\partial t}\left(\frac{n B}{n-1}\right)+u_{j} \frac{\partial}{\partial x_{j}}\left(\frac{n B}{n-1}\right)=0 .
$$

This approach was proposed by Abgrall [3] for gases and extended to the stiffened equation of state by Shyue [4]. If needed, mass and/or volume fraction can be computed from the expressions in $\$ 2.3 .2$, as long as there are only two components with different $n$. It requires additional transport equations for different material properties in the equation of state [e.g., 32].

- Volume fraction approach (five-equations model): Rather than solving transport equations for each of the material properties, Eq. (16) is rewritten in terms of the volume fraction (see Appendix A):

$$
\begin{aligned}
& \frac{\partial \rho^{(k)} \alpha^{(k)}}{\partial t}+\frac{\partial}{\partial x_{j}}\left(\rho^{(k)} \alpha^{(k)} u_{j}\right)=0, \\
& \frac{\partial \alpha^{(k)}}{\partial t}+u_{j} \frac{\partial \alpha^{(k)}}{\partial x_{j}}=\Gamma_{k k^{\prime}} \frac{\partial u_{j}}{\partial x_{j}}, \quad \Gamma_{k k^{\prime}}=\alpha^{(k)} \alpha^{\left(k^{\prime}\right)} \frac{\rho^{\left(k^{\prime}\right)}\left(a^{\left(k^{\prime}\right)}\right)^{2}-\rho^{(k)}\left(a^{(k)}\right)^{2}}{\alpha^{(k)} \rho^{\left(k^{\prime}\right)}\left(a^{\left(k^{\prime}\right)}\right)^{2}+\alpha^{\left(k^{\prime}\right)} \rho^{(k)}\left(a^{(k)}\right)^{2}}
\end{aligned}
$$

where $a^{(k)}$ is the sound speed in phase/fluid $k$. This approach written as such was introduced by Murrone \& Guillard [23] and is not restricted to the stiffened equation of state. Eq. [19b) is required to prevent pressure oscillations. For sharp-interface problems, $\Gamma_{k k^{\prime}}$ is commonly set to zero [19, 21, 33], which corresponds to the limit of infinite resolution. We also follow this convention.

- Mass fraction approach: Eq. (20) is solved,

$$
\frac{\partial z^{(k)}}{\partial t}+u_{j} \frac{\partial z^{(k)}}{\partial x_{j}}=0
$$


This approach was introduced by Shyue [4]; an alternate form of Eq. (77) to relate $z$ to $\gamma$ (for gases) in which it is implicitly assumed $M_{1}=M_{2}$ is necessary to prevent pressure oscillations, which is not true in general. This approach is not restricted to the stiffened equation of state, and only one transport equation is needed for each extra component/phase.

Although these approaches prevent the generation of pressure errors, they do not necessarily maintain temperature equilibrium. In the case of gases for instance, Johnsen \& Ham [16] showed that the mass fraction approach must be modified to prevent temperature errors. This issue is not problematic for Euler simulations since temperature is a derived quantity that does not enter the equations; however, they matter for Navier-Stokes simulations, as failure to maintain temperature equilibrium generates errors in the energy (and thus pressure) through the heat conduction term, which then affect all variables. In the next section, we identify the origin of temperature errors in gas/liquid flows and discuss how to eliminate them.

\section{Analysis of the temperature errors}

\subsection{Occurrence of temperature errors}

To illustrate the occurrence of temperature errors, we consider the $1 \mathrm{D}$ advection of an isolated material interface between a gas and a liquid at a constant velocity, pressure, and temperature, inspired by the analysis of Johnsen \& Ham [16]. Initially, $p, u$ and $T$ are constant, and $\rho$ and the material properties entering the equation of state are discontinuous. The exact solution for this problem is that this discontinuous front propagates at speed $u$; from the computational viewpoint, although the initially discontinuous profiles diffuse numerically, $p, u$ and $T$ are expected to remain constant (to round-off). We start with the compressible Navier-Stokes equations (1) and discretize them spatially using any of the three approaches in 33 Since these approaches are all designed to preserve velocity and pressure equilibrium across the interface with no heat transfer, the continuity and momentum equations demonstrate that velocity equilibrium is preserved. The energy equation simplifies to the following semi-discrete form, with the interface lying in cell $j$ :

$$
\frac{d(\rho e)}{d t}=-u D_{j}^{a}(\rho e)+D_{j}^{d}\left(\kappa D_{j}^{d}(T)\right),
$$

where $D_{j}^{a}$ and $D_{j}^{d}$ are spatial difference operators for advection and diffusion that are assumed to have the following properties [16]:

$$
D_{j}(A+c B)=D_{j}(A)+c D_{j}(B)
$$

for $c$ constant, $A$ and $B$ variable; such properties are not trivial but can be enforced, even with high-order

methods [10, 13]. Substituting the stiffened equation of state (3) into Eq. (21) yields for the pressure (assuming $\rho q$ is treated appropriately, as described in the next section):

$$
p \frac{d}{d t}\left(\frac{1}{n-1}\right)_{j}+\frac{d}{d t}\left(\frac{n B}{n-1}+\rho q\right)_{j}=-u p D_{j}^{a}\left(\frac{1}{n-1}\right)-u D_{j}^{a}\left(\frac{n B}{n-1}+\rho q\right)+D_{j}^{d}\left(\kappa D_{j}^{d}(T)\right) .
$$


Pressure equilibrium (i.e., the pressure at the next time step remains the same across the entire domain) is maintained if (i) the difference operators obey Eq. (22), (ii) the material properties entering the equation of state are evolved in a consistent fashion and (iii) the pressure is computed from the appropriate quantities 3, 4, 10]. Similarly, for the temperature:

$$
\frac{d}{d t}(\rho c T+B+\rho q)_{j}=-u T D_{j}^{a}(\rho c)-u D_{j}^{a}(B)-u D_{j}^{a}(\rho q)+D_{j}^{d}\left(\kappa D_{j}^{d}(T)\right) .
$$

Temperature equilibrium (i.e., the temperature at the next time step remains the same across the entire domain) is maintained if (i) the difference operators obey Eq. (22), (ii) the material properties entering the equation of state are evolved in a consistent fashion and (iii) the temperature is computed from the appropriate quantities. Following the results in the previous section, the difference operator for diffusion must be constructed such that $D_{j}(c)=0$ for $c$ constant [e.g., see 16]. There are thus two main sources for temperature errors: spatial discretization (i) and numerical model (ii and iii). A failure to maintain temperature equilibrium produces energy (and thus pressure) errors via the heat diffusion term in Eq. (23), which then affect the continuity and momentum equations. This issue is relevant only to problems in which heat diffusion is present.

\subsection{Eliminating temperature errors for the different approaches}

The goal is to determine the form of the transport equations to be solved and the appropriate relationships between quantities of interest to maintain temperature equilibrium for this isolated interface advection problem based on the approaches described in 93

\subsection{1. $\gamma$-based approach}

The pressure-internal energy relation (23) can be re-written:

$$
\left[\frac{d}{d t}\left(\frac{1}{n-1} p\right)_{j}+u p D_{j}^{a}\left(\frac{1}{n-1}\right)\right]+\left[\frac{d}{d t}\left(\frac{n B}{n-1}\right)_{j}+u D_{j}^{a}\left(\frac{n B}{n-1}\right)\right]+\left[\frac{d}{d t}(\rho q)_{j}+D_{j}^{a}(\rho q u)\right]=0 .
$$

Similarly, the temperature-internal energy relation (24) can be re-written:

$$
\left[\frac{d}{d t}(\rho c T)_{j}+T D_{j}^{a}(\rho c u)\right]+\left[\frac{d B_{j}}{d t}+u D_{j}^{a}(B)\right]+\left[\frac{d}{d t}(\rho q)_{j}+D_{j}^{a}(\rho q u)\right]=0 .
$$

Eqs. (25) and (26) hold for any material, pressure and temperature, therefore each term in brackets must be zero for the pressure and temperature equilibria to be maintained, in which case $p$ and $T$ can be factored out of the time derivative in Eqs. (25) and (26). Thus,

$$
\frac{d}{d t}\left(\begin{array}{c}
1 /(n-1) \\
n B /(n-1) \\
B
\end{array}\right)+u D_{j}^{a}\left(\begin{array}{c}
1 /(n-1) \\
n B /(n-1) \\
B
\end{array}\right)=\mathbf{0}, \quad \text { and } \quad \frac{d}{d t}\left(\begin{array}{c}
\rho q \\
\rho c
\end{array}\right)+D_{j}^{a}\left(\begin{array}{c}
\rho u q \\
\rho u c
\end{array}\right)=\mathbf{0} .
$$


Eqs. (27) indicate that, to maintain pressure equilibrium in time and space with the $\gamma$-based approach, the transport equations for $1 /(n-1)$ and $n B /(n-1)$ must be solved in non-conservative form and that for $q$ in conservative form; pressure must be computed from these specific quantities using Eq. (3a). To maintain temperature equilibrium, the transport equations for $B$ must be solved in non-conservative form and those for $c$ and $q$ in conservative form; temperature must be computed from these specific quantities in Eq. (3b).

\subsubsection{Volume fraction approach}

Considering the mixture relations for volume fraction (11), the pressure-internal energy relation (23) can be re-written:

$$
\begin{aligned}
& {\left[\frac{d}{d t}\left(\sum_{k} \alpha^{(k)} \frac{1}{\left(n^{(k)}-1\right)} p\right)_{j}+u p D_{j}^{a}\left(\sum_{k} \alpha^{(k)} \frac{1}{\left(n^{(k)}-1\right)}\right)\right]+\left[\frac{d}{d t}\left(\sum_{k} \alpha^{(k)} \frac{n^{(k)} B^{(k)}}{\left(n^{(k)}-1\right)}\right)_{j}+\right.} \\
& \left.u D_{j}^{a}\left(\sum_{k} \alpha^{(k)} \frac{n^{(k)} B^{(k)}}{\left(n^{(k)}-1\right)}\right)\right]+\left[\frac{d}{d t}\left(\sum_{k} \rho^{(k)} \alpha^{(k)} q^{(k)}\right)_{j}+D_{j}^{a}\left(\sum_{k} \rho^{(k)} \alpha^{(k)} q^{(k)} u\right)\right]=0 .
\end{aligned}
$$

Likewise, the temperature-internal energy relation (24) can be expressed as:

$$
\begin{aligned}
& {\left[\frac{d}{d t}\left(\sum_{k} \rho^{(k)} \alpha^{(k)} c^{(k)} T\right)_{j}+T D_{j}^{a}\left(\sum_{k} \rho^{(k)} \alpha^{(k)} c^{(k)} u\right)\right]+\left[\frac{d}{d t}\left(\sum_{k} \alpha^{(k)} B^{(k)}\right)_{j}+\right.} \\
& \left.u D_{j}^{a}\left(\sum_{k} \alpha^{(k)} B^{(k)}\right)\right]+\left[\frac{d}{d t}\left(\sum_{k} \rho^{(k)} \alpha^{(k)} q^{(k)}\right)_{j}+D_{j}^{a}\left(\sum_{k} \rho^{(k)} \alpha^{(k)} q^{(k)} u\right)\right]=0 .
\end{aligned}
$$

Since pressure and temperature must remain constant in time and space, arguments similar to those made in the previous section hold, so that $p$ and $T$ can be factored out of Eqs. (28) and (29). Thus,

$$
\begin{aligned}
\frac{d}{d t}\left(\begin{array}{c}
\sum_{k} \alpha^{(k)} /\left(n^{(k)}-1\right) \\
\sum_{k} \alpha^{(k)} n^{(k)} B^{(k)} /\left(n^{(k)}-1\right) \\
\sum_{k} \alpha^{(k)} B^{(k)}
\end{array}\right)+u D_{j}^{a}\left(\begin{array}{c}
\sum_{k} \alpha^{(k)} /\left(n^{(k)}-1\right) \\
\sum_{k} \alpha^{(k)} n^{(k)} B^{(k)} /\left(n^{(k)}-1\right) \\
\sum_{k} \alpha^{(k)} B^{(k)}
\end{array}\right) & =\mathbf{0}, \\
\frac{d}{d t}\left(\begin{array}{c}
\sum_{k} \rho^{(k)} \alpha^{(k)} q^{(k)} \\
\sum_{k} \rho^{(k)} \alpha^{(k)} c^{(k)}
\end{array}\right)+D_{j}^{a}\left(\begin{array}{c}
\sum_{k} \rho^{(k)} \alpha^{(k)} q^{(k)} \\
\sum_{k} \rho^{(k)} \alpha^{(k)} c^{(k)}
\end{array}\right) & =\mathbf{0 .}
\end{aligned}
$$

Since only mixture density, species density and volume fraction vary in time and space, Eqs.(30) are discretizations of the following two transport equations for volume fraction:

$$
\begin{aligned}
\frac{\partial\left(\rho^{(k)} \alpha^{(k)}\right)}{\partial t}+\frac{\partial}{\partial x}\left(\rho^{(k)} \alpha^{(k)} u\right) & =0, \\
\frac{\partial \alpha^{(k)}}{\partial t}+u \frac{\partial \alpha^{(k)}}{\partial x} & =0 .
\end{aligned}
$$

Eqs. (31) indicate that, to maintain pressure equilibrium in time and space for the volume fraction approach, the calculation of $1 /(n-1)$ and $n B /(n-1)$ in Eq. (10) must be done using volume fraction computed from the 
non-conservative form of the transport equation for $\alpha^{(k)}$, and that of $\rho q$ in Eq. (10) using the conservative form of the transport equation for $\alpha^{(k)}$. To maintain temperature equilibrium in time and space, the

\subsubsection{Mass fraction approach}

Using the mixture relations for mass fraction (15), the pressure-internal energy Eq. (23) can be re-written:

$$
\begin{aligned}
& {\left[\frac{d}{d t}\left(\sum_{k} z^{(k)} \frac{\rho}{\rho^{(k)}\left(n^{(k)}-1\right)} p\right)_{j}+u D_{j}^{a}\left(\sum_{k} z^{(k)} \frac{\rho}{\rho^{(k)}\left(n^{(k)}-1\right)} p\right)\right]+\left[\frac{d}{d t}\left(\sum_{k} z^{(k)} \frac{n^{(k)} B^{(k)} \rho}{\rho^{(k)}\left(n^{(k)}-1\right)}\right)_{j}+\right.} \\
& \left.u D_{j}^{a}\left(\sum_{k} z^{(k)} \frac{n^{(k)} B^{(k)} \rho}{\rho^{(k)}\left(n^{(k)}-1\right)}\right)\right]+\left[\frac{d}{d t}\left(\sum_{k} \rho z^{(k)} q^{(k)}\right)_{j}+D_{j}^{a}\left(\sum_{k} \rho z^{(k)} q^{(k)} u\right)\right]=0 .
\end{aligned}
$$

Similarly, the temperature-internal energy relation (24) can be re-written:

$$
\begin{aligned}
& {\left[\frac{d}{d t}\left(\sum_{k} \rho z^{(k)} c^{(k)} T\right)_{j}+T D_{j}^{a}\left(\sum_{k} \rho z^{(k)} c^{(k)} u\right)\right]+\left[\frac{d}{d t}\left(\sum_{k} z^{(k)} \frac{B^{(k)} \rho}{\rho^{(k)}}\right)_{j}+u D_{j}^{a}\left(\sum_{k} z^{(k)} \frac{B^{(k)} \rho}{\rho^{(k)}}\right)\right]} \\
& +\left[\frac{d}{d t}\left(\sum_{k} \rho z^{(k)} q^{(k)}\right)_{j}+D_{j}^{a}\left(\sum_{k} \rho z^{(k)} q^{(k)} u\right)\right]=0 .
\end{aligned}
$$

Following the same arguments as in the previous sections, Eqs. (32) and (33) can be re-organized:

$$
\begin{aligned}
& \frac{d}{d t}\left(\begin{array}{c}
\sum_{k} \rho z^{(k)} /\left[\rho^{(k)}\left(n^{(k)}-1\right)\right] \\
\sum_{k} \rho z^{(k)} n^{(k)} B^{(k)} /\left[\rho^{(k)}\left(n^{(k)}-1\right)\right] \\
\sum_{k} \rho z^{(k)} B^{(k)} / \rho^{(k)}
\end{array}\right)+u D_{j}^{a}\left(\begin{array}{c}
\sum_{k} \rho z^{(k)} /\left[\rho^{(k)}\left(n^{(k)}-1\right)\right] \\
\sum_{k} \rho z^{(k)} n^{(k)} B^{(k)} /\left[\rho^{(k)}\left(n^{(k)}-1\right)\right] \\
\sum_{k} \rho z^{(k)} B^{(k)} / \rho^{(k)}
\end{array}\right)=\mathbf{0}, \\
& \frac{d}{d t}\left(\begin{array}{c}
\sum_{k} \rho z^{(k)} q^{(k)} \\
\sum_{k} \rho z^{(k)} c^{(k)}
\end{array}\right)+D_{j}^{a}\left(\begin{array}{c}
\sum_{k} \rho z^{(k)} q^{(k)} \\
\sum_{k} \rho z^{(k)} c^{(k)}
\end{array}\right)=\mathbf{0}
\end{aligned}
$$


Since only mixture density, species density, and species mass fraction vary in time and space, Eqs.(34) are discretizations of the following two transport equations for mass fraction:

$$
\begin{aligned}
\frac{\partial}{\partial t}\left(\rho z^{(k)}\right)+\frac{\partial}{\partial x}\left(\rho z^{(k)} u\right) & =0, \\
\frac{\partial}{\partial t}\left(\frac{\rho}{\rho^{(k)}} z^{(k)}\right)+u \frac{\partial}{\partial x}\left(\frac{\rho}{\rho^{(k)}} z^{(k)}\right) & =0 .
\end{aligned}
$$

From Eq. (6), Eqs. (35) are mathematically equivalent to those for volume fraction, such that the same conditions as those listed in 4.2 .2 hold to maintain pressure and temperature equilibria.

\subsection{Summary of the analysis}

The analysis in the previous section indicates that all three approaches $(\gamma$, volume fraction and mass fraction) can be designed to prevent pressure and temperature errors. For two fluids, the $\gamma$-based approach is computationally more expensive because one transport equation must be solved for each property in the equation of state (five here); for more than two fluids it may become more attractive, but if fluids have the same properties additional transport equations must be solved to distinguish the different fluids.

The volume fraction and mass fraction approaches are mathematically equivalent. These approaches are not tailored to a given equation of state; however, analysis is required to determine how to calculate the material properties entering the equation of state. For each additional fluid, two additional transport equations must be computed. The reduced five-equation model (with $\Gamma_{k k^{\prime}}=0$ ) is strictly applicable only to flows of immiscible fluids (no physical mixture regions); for miscible flows, $\Gamma_{k k^{\prime}} \neq 0$ and the source term in the transport equation must be computed, which may lead to difficulties with shock jump conditions and positivity of volume fraction. These approaches can be extended to more general equations of state, such as Mie-Grüneisen [e.g., see 6].

\section{Numerical implementation}

For the simulations presented in this work, time marching is handled with a third-order accurate explicit strong stability preserving Runge-Kutta scheme [37]. For the spatial discretization, a solution-adaptive highorder accurate central difference/discontinuity-capturing method is proposed. This method can represent both broadband flow motions and discontinuities accurately and efficiently. The basic idea is that nondissipative methods are used where the solution is smooth, while the more dissipative and computationally expensive capturing schemes are applied near discontinuous regions. For this purpose, a discontinuity sensor discriminates between smooth and discontinuous (shocks, contacts and interfaces) regions, which all require a different treatment; smooth regions are computed using central differences, a finite difference weighted essentially non-oscillatory [WENO 38 scheme with Lax-Friedrichs flux splitting handles shock waves, and the approach of Johnsen \& Colonius [10] is used for material interfaces. 
To illustrate the specifics, we consider the semi-discrete form of the 1D Euler equations for simplicity,

$$
\frac{d}{d t} u+\frac{F_{i+1 / 2}-F_{i-1 / 2}}{\Delta x}=0
$$

where $u$ is the vector of conserved variables and $F$ is the numerical flux, which can be written

$$
F_{i+1 / 2}=\left.b_{1} F_{i+\frac{1}{2}}\right|_{\text {central }}+\left.b_{2} F_{i+\frac{1}{2}}\right|_{\text {shock }}+\left.b_{3} F_{i+\frac{1}{2}}\right|_{\text {int }},
$$

where $b_{i}$ represent the value of the sensor in different regions. The sensor values are $b_{1}=1$ and $b_{2}, b_{3}=0$ for smooth regions, $b_{2}=1$ and $b_{1}, b_{3}=0$ for shocks and $b_{3}=1$ and $b_{1}, b_{2}=0$ for interfaces. The capability of the sensor to distinguish between discontinuous and smooth regions highly affects the overall accuracy and performance [39]. We adapt the sensor of Henry de Frahan et al. 6] to finite differences. At each cell edge, $L$ and $R$ denote the value of the corresponding variable at the left and right of the computational cell respectively. Accordingly, shocks are detected using the function below:

$$
\phi=\frac{p_{R}-p_{L}}{p_{R}+p_{L}}, \quad \Phi=\frac{2 \phi}{(1+\phi)^{2}} .
$$

If $\Phi$ is greater than 0.01 , the corresponding cell is flagged to be treated by shock capturing. Contact discontinuities, also treated by shock capturing, are detected as follows:

$$
\Xi=\frac{2 \xi}{(1+\xi)^{2}}, \quad \xi=\frac{\rho_{R}-\rho_{L}}{\rho_{R}+\rho_{L}} .
$$

Finally, material interfaces, to be handled with the method of Johnsen \& Colonius [10] are detected as follows:

$$
Z=\frac{2 \zeta}{(1+\zeta)^{2}}, \quad \zeta=\frac{n_{R}-n_{L}}{n_{R}+n_{L}}
$$

The thresholds for $\Xi$ or $Z$ are set to be 0.01 . Although the optimal threshold values for both shocks and interfaces may be problem-dependent, our numerical experiments show a robust detection of discontinuities for the chosen values, which are slightly different from those in Henry de Frahan et al. 6] because of the different spatial discretization. This approach is easily applicable to multiple dimensions.

In smooth regions, fourth-order central differences are used for the convective terms, both for the conservative and non-conservative equations as proposed by Movahed \& Johnsen [5]:

$$
F_{i+1 / 2}^{\text {Central }}=\frac{1}{12}\left(-F_{i+2}+7 F_{i+1}+7 F_{i}-F_{i-1}\right) .
$$

Second derivatives (e.g., for diffusion) are also treated with fourth-order differences; for an arbitrary variable $A$,

$$
\left.A_{x x}\right|_{i}=\frac{-A_{i+2}+16 A_{i+1}-30 A_{i}+16 A_{i-1}-A_{i-2}}{12 \Delta x^{2}} .
$$

Shock waves are captured using the fifth-order accurate finite difference WENO of Jiang \& Shu [38]. For this purpose, we use Lax-Friedrichs flux splitting,

$$
F^{ \pm}(u)=\frac{1}{2}(F(u) \pm \lambda u), \quad \text { with } F_{i+1 / 2}^{L X F}=\hat{F}_{i+1 / 2}^{+}+\hat{F}_{i+1 / 2}^{-} .
$$


where $\lambda=\max _{u}\left|F^{\prime}(u)\right|$ over the relevant range of $u$. Since these regions are not flagged as material discontinuities, the central scheme can be used to solve the advection equation for the (constant) material properties.

For material interfaces, the WENO procedure proposed by Johnsen \& Colonius [10] is used, which corresponds to a second-order finite difference approximation; however, this reduction in order is not problematic since this approach is used at material discontinuities only, where the solution reduces to first order anyways. An HLL Riemann solver [40] is used for upwinding. To correctly treat the non-conservative transport equations, we extend the expression in Saurel \& Abgrall [41] to high-order WENO:

$$
F_{i+1 / 2}^{H L L}=\frac{S^{+} F_{i+1 / 2}^{L}-S^{-} F_{i+1 / 2}^{R}+S^{+} S^{-}\left(U_{i+1 / 2}^{R}-U_{i+1 / 2}^{L}\right)}{S^{+}-S^{-}},
$$

where $U_{i+1 / 2}^{R}$ and $U_{i+1 / 2}^{L}$ are the reconstructed variables on the right and left of cell $i+1 / 2, F_{i+1 / 2}^{R}$ and $F_{i+1 / 2}^{L}$ are the corresponding fluxes, and $S^{+}$and $S^{-}$are the right and left wave speed, respectively, calculated from:

$$
S^{+}=\max \left(0, u_{i+1 / 2}^{R}+a_{i+1 / 2}^{R}, u_{i+1 / 2}^{L}+a_{i+1 / 2}^{L}\right), \quad S^{-}=\min \left(0, u_{i+1 / 2}^{R}-a_{i+1 / 2}^{R}, u_{i+1 / 2}^{L}-a_{i+1 / 2}^{L}\right),
$$

where $u$ and $a$ stand for velocity and sound speed, respectively. The discretized form of the non-conservative transport equation for an arbitrary variable $A$ is

$$
\begin{aligned}
& A_{i}^{n+1}=A_{i}^{n}-\frac{\Delta t}{\Delta x}\left[\frac{u_{i}^{n}\left(S_{i+1 / 2}^{+} A_{i+1 / 2, R}^{n}-S_{i+1 / 2}^{-} A_{i+1 / 2, L}^{n}\right)+S_{i+1 / 2}^{+} S_{i+1 / 2}^{-}\left(A_{i+1 / 2, R}^{n}-A_{i+1 / 2, L}^{n}\right)}{S_{i+1 / 2}^{+}-S_{i+1 / 2}^{-}}\right. \\
& \left.-\frac{u_{i}^{n}\left(S_{i-1 / 2}^{+} A_{i-1 / 2, R}^{n}-S_{i-1 / 2}^{-} A_{i-1 / 2, L}^{n}\right)+S_{i-1 / 2}^{+} S_{i-1 / 2}^{-}\left(A_{i-1 / 2, R}^{n}-A_{i-1 / 2, L}^{n}\right)}{S_{i-1 / 2}^{+}-S_{i-1 / 2}^{-}}\right] .
\end{aligned}
$$

\section{Results}

The compressible Navier-Stokes equations, non-dimensionalized by the density and sound speed of air at atmospheric pressure, characteristic length $L=0.2 \mathrm{~mm}$, and $T=300 \mathrm{~K}$, are solved for all problems. The time step is adaptively set to satisfy the advection and diffusion constraints, with CFL number 0.95 and VNN 0.475 . We consider the $\gamma$, volume fraction (or " $\alpha$ ") and mass fraction (or " $z$ ") models described in 4.2 when referring to our proposed approach, we mean an approach that preserves velocity, pressure and temperature equilibria (for an isolated interface). We make comparisons to current schemes in the literature designed to maintain velocity and pressure equilibria in the absence of heat conduction, which we call "pressure only" [e.g., the methods in 3, 4]; with these approaches, temperature is computed from the available data; e.g., for the $\gamma$-model, $B$ would be computed from $n B /(n-1)$ and $1 /(n-1)$, rather than being advected as we propose. The $\alpha$ and $z$ "pressure only" approaches are identical, so only the $z$ approach is considered. Water and air have values taken from Table 1 


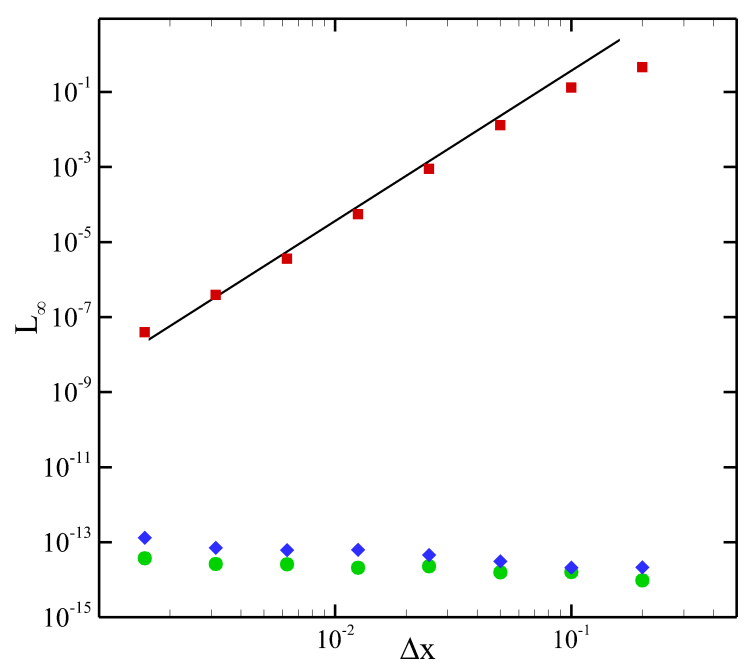

Figure 1: $L_{\infty}$ error for the 1D smooth advection problem. Red squares: $n$; blue diamonds: pressure; green circles: temperature.

\subsection{D smooth advection problem}

We consider the advection of a smooth distribution in density and $n$ to show that our solution-adaptive method achieves the correct convergence rate for smooth problems. The following initial conditions are used

$$
(\rho, u, p, T, n)=(1+0.2 \sin (4 \pi x), 1,1,1,1.4+0.2 \sin (\pi x)) .
$$

This distribution moves at constant speed $u$, with constant pressure and temperature in the periodic domain $x \in[0,1]$. The $L_{\infty}$ errors in $n$, pressure and temperature are shown in Fig. (11) after one period and for different resolutions. Pressures and temperatures remain near round-off, thus demonstrating that pressure and temperature equilibria are maintained. The convergence rate (in $n$ ) is fourth, the order of the finite difference scheme.

\section{2. $1 D$ air/water interface advection}

We numerically verify our theoretical development for the isolated interface advection problem in 4.1 e.g., for a $1 \mathrm{D}$ air bubble in water. We consider an initially sharp top-hat distribution of air in water at the same temperature and pressure, moving at a constant speed $u$ in the periodic domain $x \in[0,1]$. The initial conditions are,

$$
(\rho, u, p, T)= \begin{cases}(1,0.5,0.716,1) & \text { if } x / L \in[0.25,0.75] \\ (848.28,0.5,0.716,1) & \text { otherwise }\end{cases}
$$

The properties entering the equation of state are initialized using the same top-hat distribution. The results for all three models with the "pressure only" and our proposed approaches using 200 points are shown in Figs. 2 (density, pressure and temperature profiles after one period) and 3 (time evolution of the $L_{\infty}$ error in $u, p, T)$. The results clearly indicate that our proposed approaches do not introduce errors in pressure, 


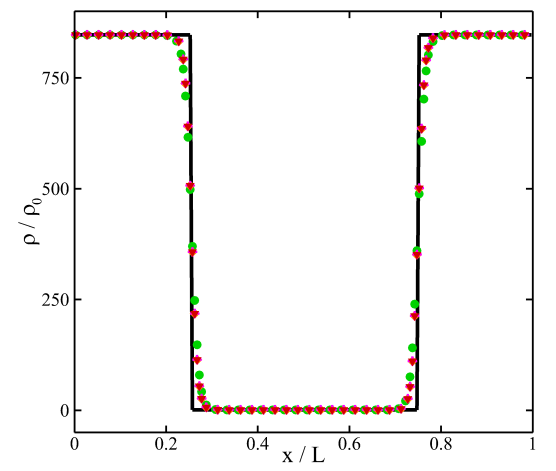

(a) Density.

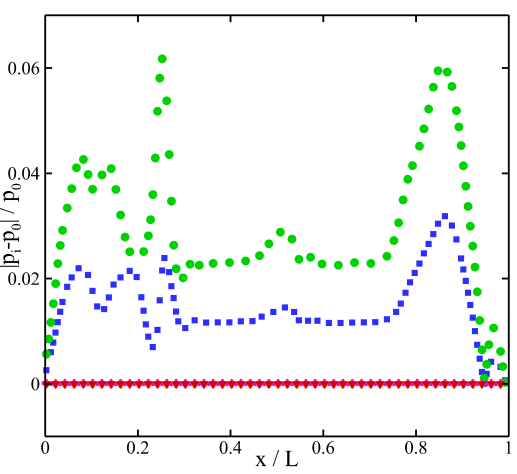

(b) Pressure.

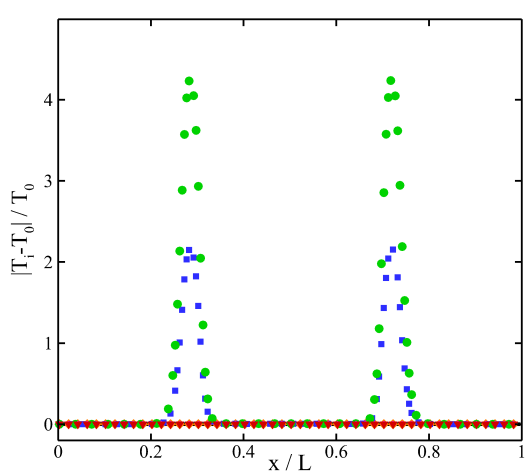

(c) Temperature.

Figure 2: Profile of the advection of an air/water interface after one period. Black solid line: initial and exact solution; green circles: "pressure only" $\gamma$ approach; blue squares: "pressure only" $z$ approach; pink pluses: proposed $\gamma$ approach; red triangles: proposed $\alpha$ approach; orange diamonds: proposed $z$ approach.

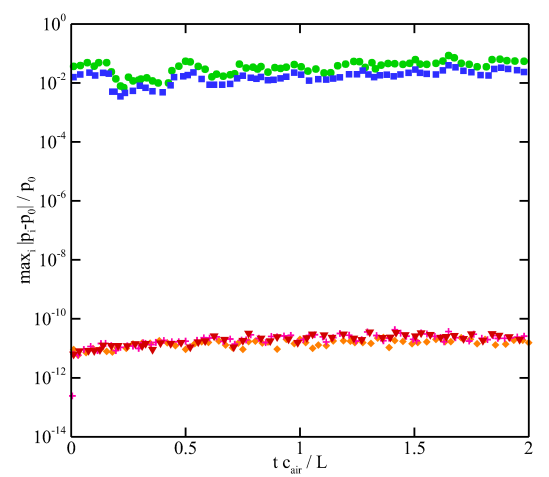

(a) Pressure

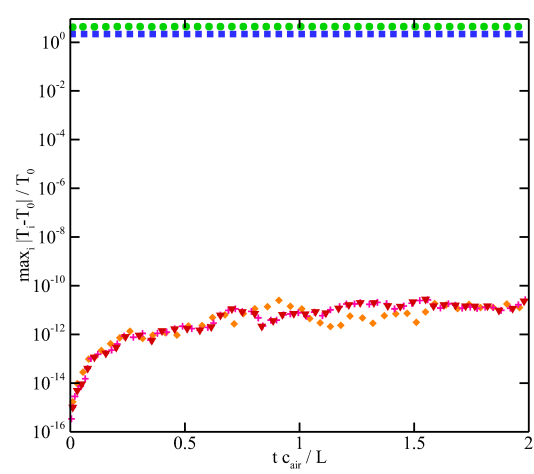

(b) Temperature

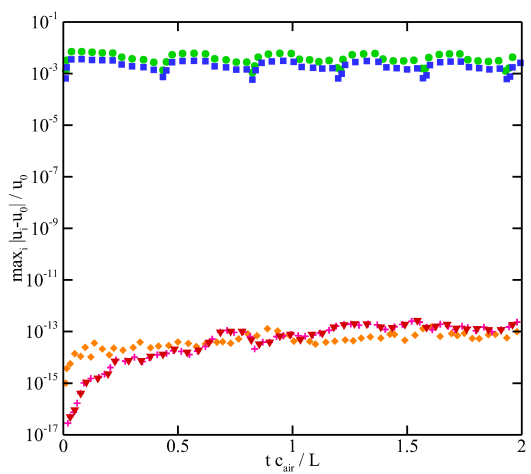

(c) Velocity

Figure 3: Time histories of normalized $L_{\infty}$ errors for the advection of an air/water interface after one period. Black solid line: initial and exact solution; green circles: "pressure only" $\gamma$ approach; blue squares: "pressure only" $z$ approach; pink pluses: proposed $\gamma$ approach; red triangles: proposed $\alpha$ approach; orange diamonds: proposed $z$ approach.

temperature or velocity. On the other hand, if using approaches that are designed to only preserve pressure and velocity equilibria, and using only the available data to compute temperature, then errors are produced in the temperature, which then propagate to the other fields due to the heat diffusion and pressure terms. The resulting errors are non-negligible, particularly for the temperature. If the Fourier heat conduction term had not been included no such errors would occur.

\subsection{D gas-liquid Riemann problem}

We consider gas-liquid Riemann problems to compare the "pressure only" and our proposed $\alpha$ approaches for shock-dominated interfacial flows; similar results are obtained with the other models. The initial condi- 
tions (with water on the left, air on the right) are [19, 23]:

$$
(\rho, u, p)= \begin{cases}(1000,0,8300) & \text { if } x / L \in[0,0.7] \\ (50,0,0.83) & \text { if } \quad x / L \in[0.7,1]\end{cases}
$$

The domain is discretized with 1,000 cells and the exact solution is the converged solution on 5,000 cells. The profiles of density, pressure, temperature, velocity, and volume fraction for both approaches are shown in Fig. (4). Our proposed approach shows good agreement with the exact solution. For the "pressure only" approach at this high pressure ratio (10,000:1) and heavy gas, temperature errors are clear at the interface, but pressure errors are not substantial.

Another gas-liquid Riemann problem is considered with initial conditions more relevant to our interests (1D shock-bubble interaction):

$$
(\rho, u, p)= \begin{cases}(881.6,0.184,714) & \text { if } x / L \in[0,0.7] \\ (1,0,0.714) & \text { if } x / L \in[0.7,1]\end{cases}
$$

The domain is discretized with 1000 cells and the exact solution is the converged solution on 5,000 cells. The profiles of density, pressure, temperature, velocity, and volume fraction for both approaches are shown in Fig. (5). The numerical solution agrees well with the exact solution. In this problem, the effect of heat diffusion is significant inside the bubble. On the other hand, the "pressure only" approach gives rise to a large temperature error at the interface, as well as erroneous density, velocity, pressure and temperature between the interface and shock.

\subsection{D shock-bubble interaction}

To determine the implications for relevant bubble dynamics problems, we consider the interaction of a shock wave in water with an air bubble near a rigid wall, as in Johnsen et al. [12]. Initially, the spherical bubble is in equilibrium with its surroundings:

$$
(\rho, u, v, w, p, T)= \begin{cases}(1,0,0,0,0.714,1) & \text { in the bubble } \\ (846.2,0,0,0,0.714,1) & \text { in the water upstream of the shock. }\end{cases}
$$

Two different shock strengths are considered:

- Case 1: weak shock wave with pressure ratio of 100 (Mach 1.0035)

$$
(\rho, u, v, w, p, T)=(849.8,0.0188,0,0,71.4,1.006) \quad \text { in the water downstream of the shock. }
$$

- Case 2: strong shock wave with pressure ratio of 1000 (Mach 1.035)

$$
(\rho, u, v, w, p, T)=(881.6,0.184,0,0,714,1.06) \quad \text { in the water downstream of the shock. }
$$




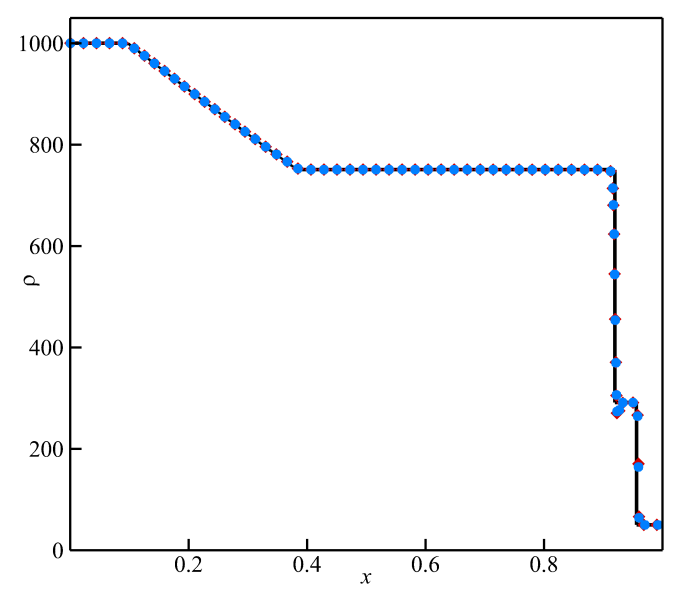

(a) Density.

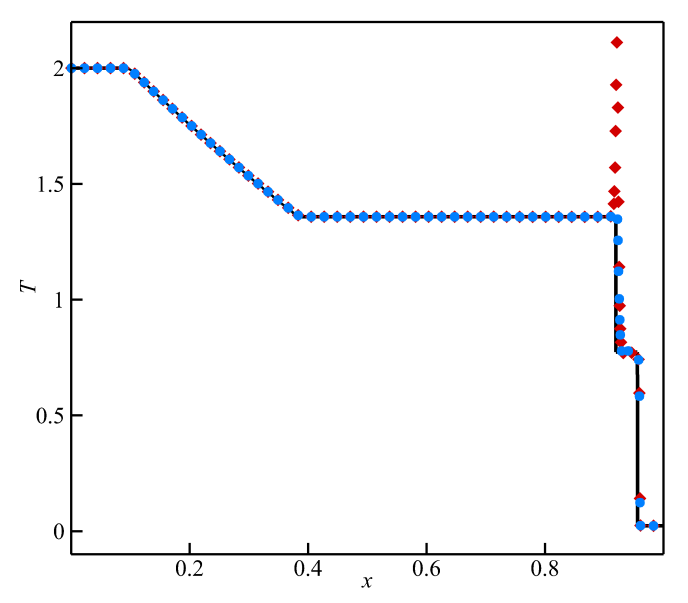

(c) Temperature.

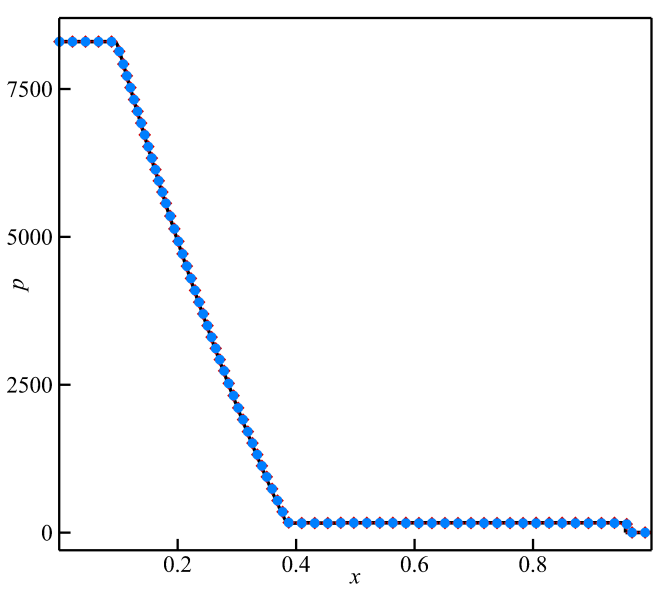

(b) Pressure.

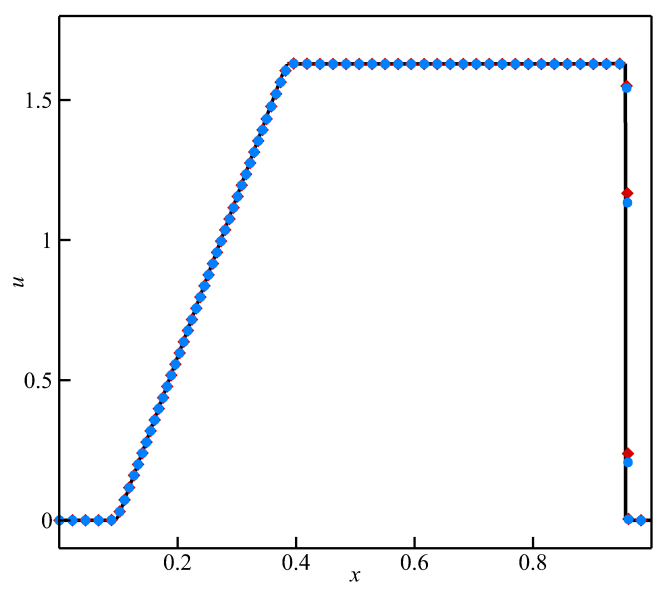

(d) Velocity.

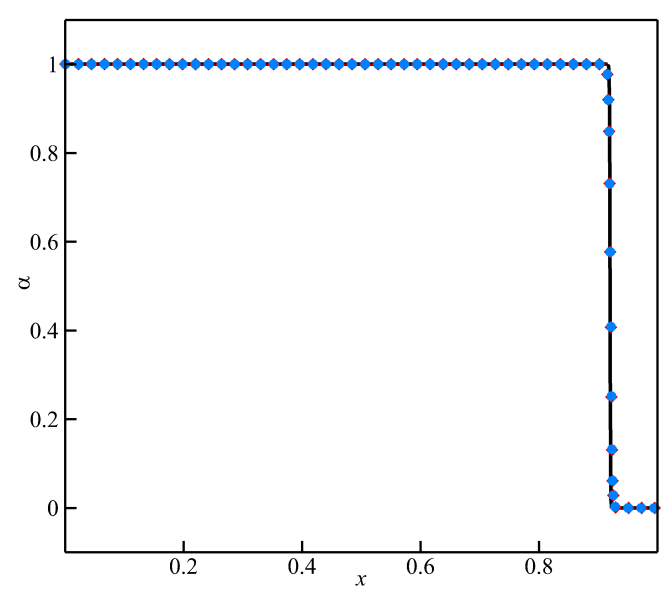

(e) Volume fraction of water.

Figure 4: Gas-liquid Riemann problem [19, 23]. Black solid line: exact solution; blue filled circles: proposed approach; red filled diamonds: "pressure only" approach. 


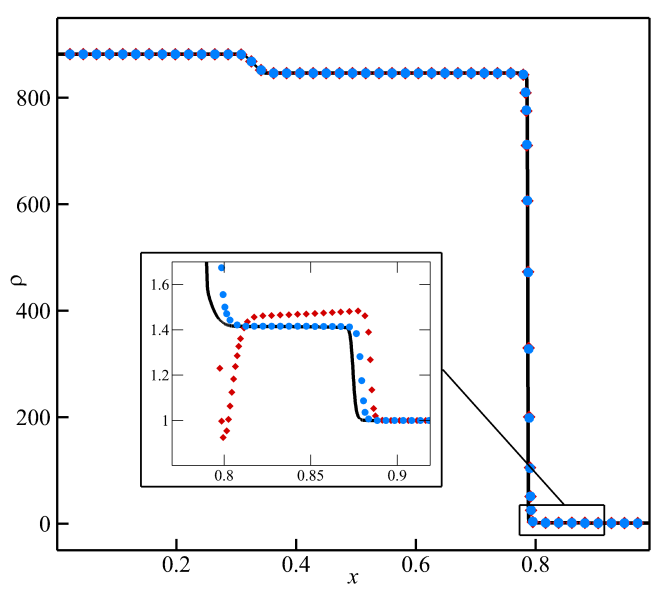

(a) Density.

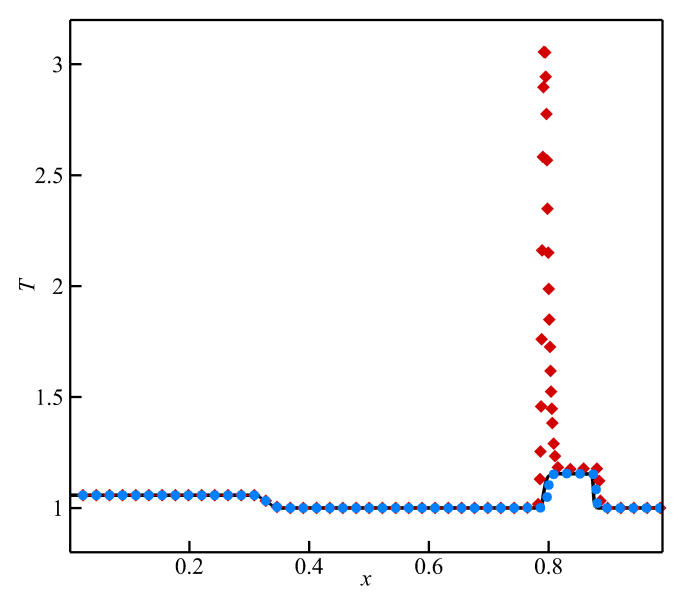

(c) Temperature.

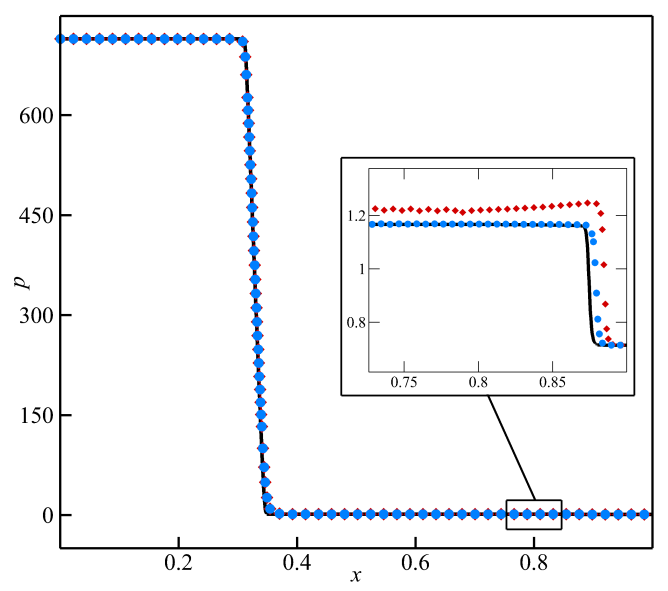

(b) Pressure.

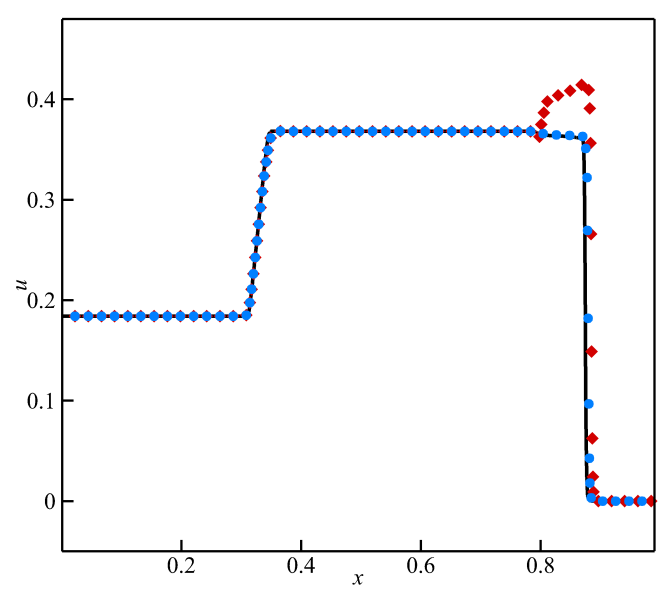

(d) Velocity.

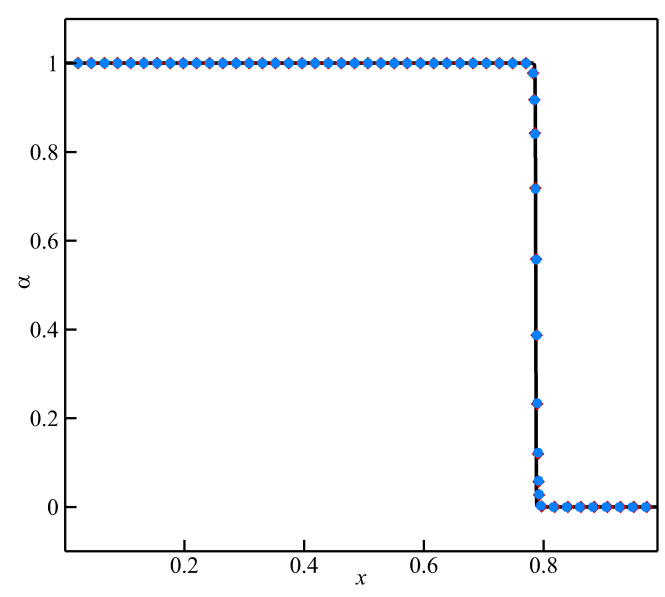

(e) Volume fraction of water.

Figure 5: Gas-liquid Riemann problem (1D shock-interface). Black solid line: exact solution; blue filled circles: proposed approach; red filled diamonds: "pressure only" approach. 

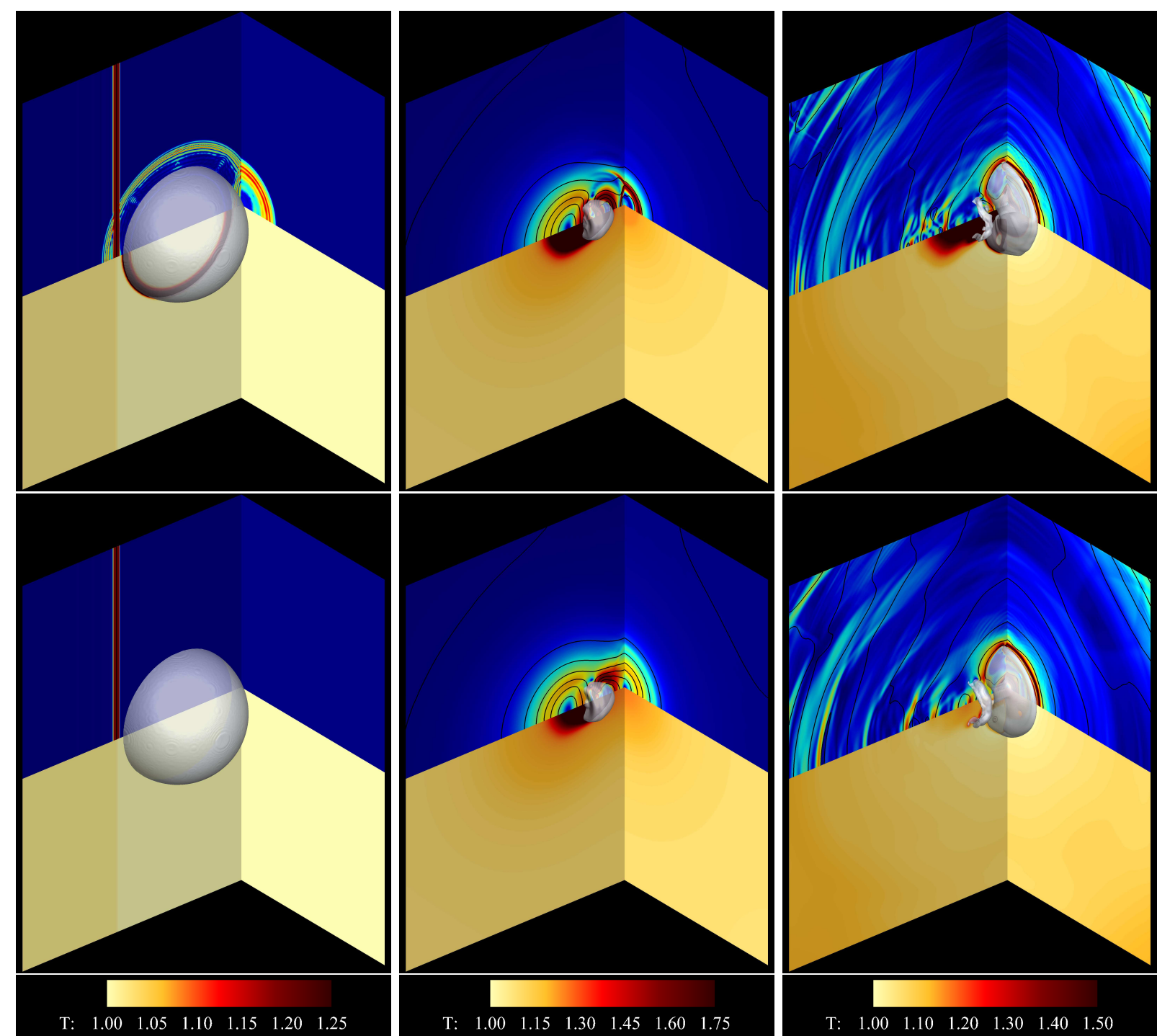

Figure 6: Shock-induced bubble collapse (case $1, p_{s} / p_{o}=100$ ) at different times $t=0.04,1.49,2.05$. top row: "pressure only" approach; bottom row: proposed approach; top contour: pressure gradient magnitude; bottom contour: temperature.

This problem is simulated using the "pressure only" and our proposed $\alpha$ approaches on a $500 \times 400 \times 400$ uniform grid for both cases. The initial stand-off distance of the bubble from the wall is 1.1. By symmetry, only a quarter of the bubble is computed, with symmetry boundary conditions along the relevant planes. The wall a purely reflecting, with no slip. Zero gradient conditions are used along the remaining boundaries.

The results are compared to evaluate the effects of temperature errors; quantities related to pressures and temperatures along the wall, as well as bubble dynamics are of particular interest. Figs. (6) and (7) show the pressure gradient magnitude and temperature contours at different times during the process. The right-moving shock interacts with the bubble, thus producing a reflected rarefaction wave. As the bubble 

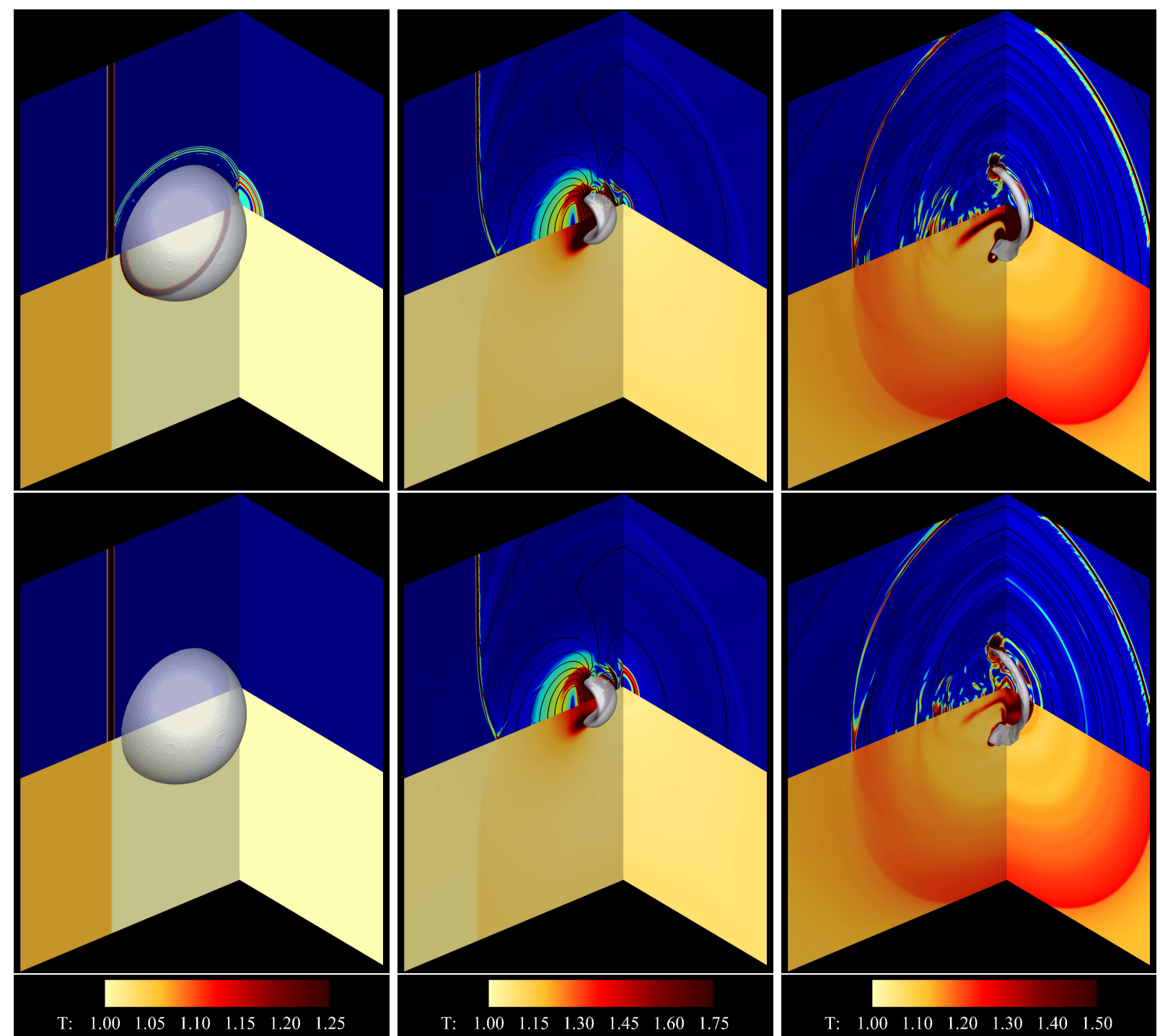

Figure 7: Shock-induced bubble collapse (case $2, p_{s} / p_{o}=1000$ ) at different times $t=0.03,0.6,0.92$. top row: "pressure only" approach; bottom row: proposed approach; top contour: pressure gradient magnitude; bottom contour: temperature.

starts its collapse, the incoming shock hits the rigid wall and reflects back onto the bubble. During the collapse, a re-entrant jet directed toward the wall is produced, which, upon impact with the distal side, generates an outward propagating shock. Even though the "pressure only" approach is designed to prevent pressure errors, such errors are generated because of the large temperature spike across the bubble interface. The most striking discrepancies lie in the temperature contours, particularly in the region just outside the bubble after collapse. The pressure gradient magnitude shows how these temperature errors propagate in the pressure field. These temperature errors strongly affect the simulations and may ultimately cause the code to fail. 

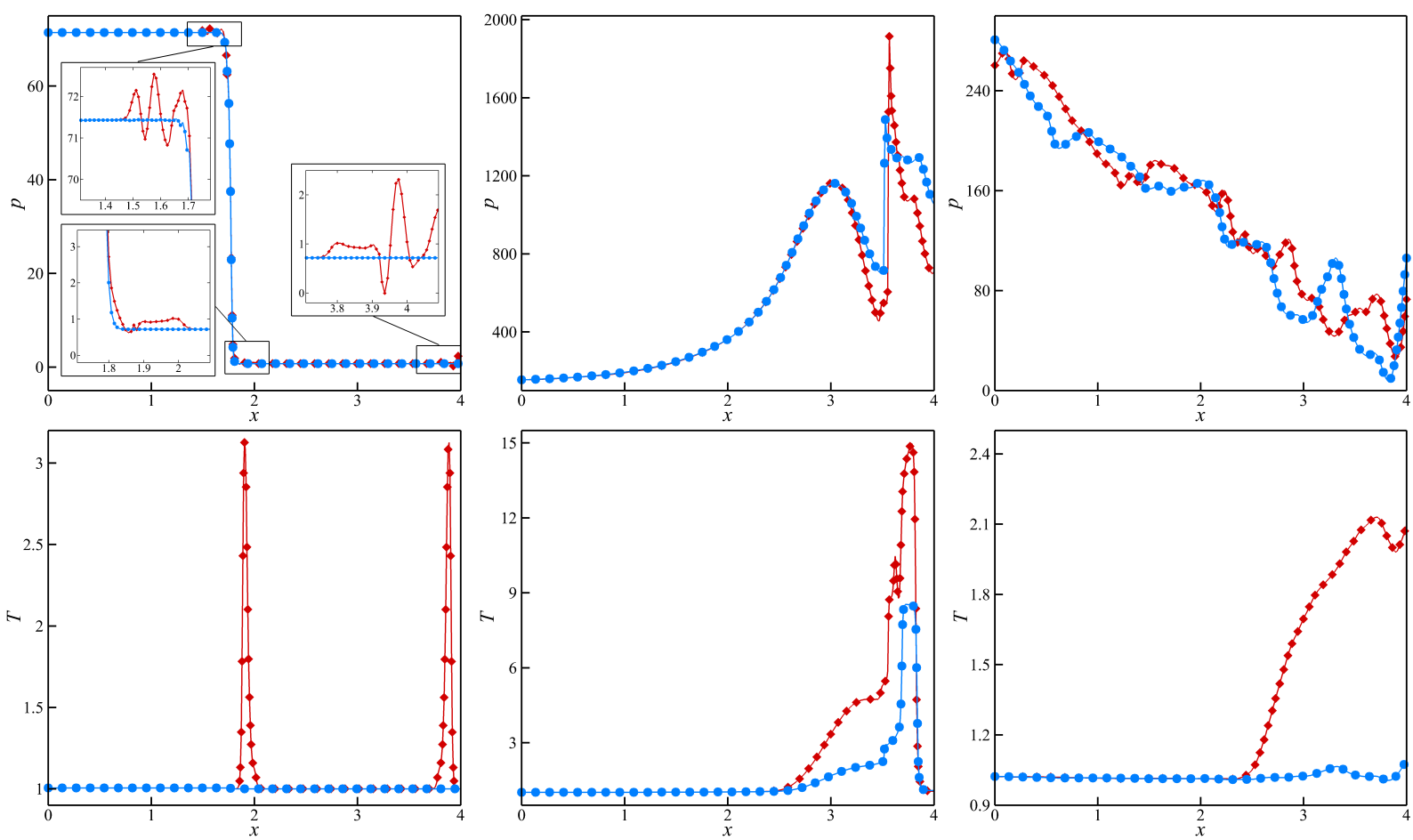

Figure 8: Shock-induced bubble collapse (case 1, $p_{s} / p_{o}=100$ ) - Centerline properties (top row: pressure; bottom row: temperature) at times $0.04,1.49,2.05$. Red diamonds: "pressure only" approach; blue circles: proposed approach.

To quantitatively evaluate these errors, Figs. (8) and (9) show the pressure and temperature along the centerline at different times, and Figs. (10) and (11) plot time histories of the wall pressure and temperature at point $A$ (along the centerline and on the wall), and total enstrophy for both cases. The temperature errors initially consist of spikes along the interface of over $100 \%$ error. Much of the temperature discrepancies appear to be localized at the interface, though some regions in which the interface was previously located appear to still bear memory of these errors. Temperature errors are particularly important for case $1\left(p_{s} / p_{o}=100\right)$. The pressure oscillations emanating from these temperature errors exhibit the largest discrepancies after collapse in the region between the bubble and the wall, with local errors nearly $100 \%$. Along the wall, the discrepancies in pressure are on the order of $10 \%$, while the temperature errors are more than $100 \%$, always overshoots: for case 1, the maximum wall pressure and temperature in the simulation for the proposed approach are 2,356 and 1.2, while the "pressure only" approaches yield 2,550 and 2.2; for case 2, the maximum wall pressure and temperature in the simulation for the proposed approach are 14,450 and 4.1, while the "pressure only" approaches yield 15,300 and 10.2. These discrepancies even affect the vorticity contents of the flow and possibly generation of small-scale features, due to baroclinic vorticity generated along the interface, due to pressure oscillations. 

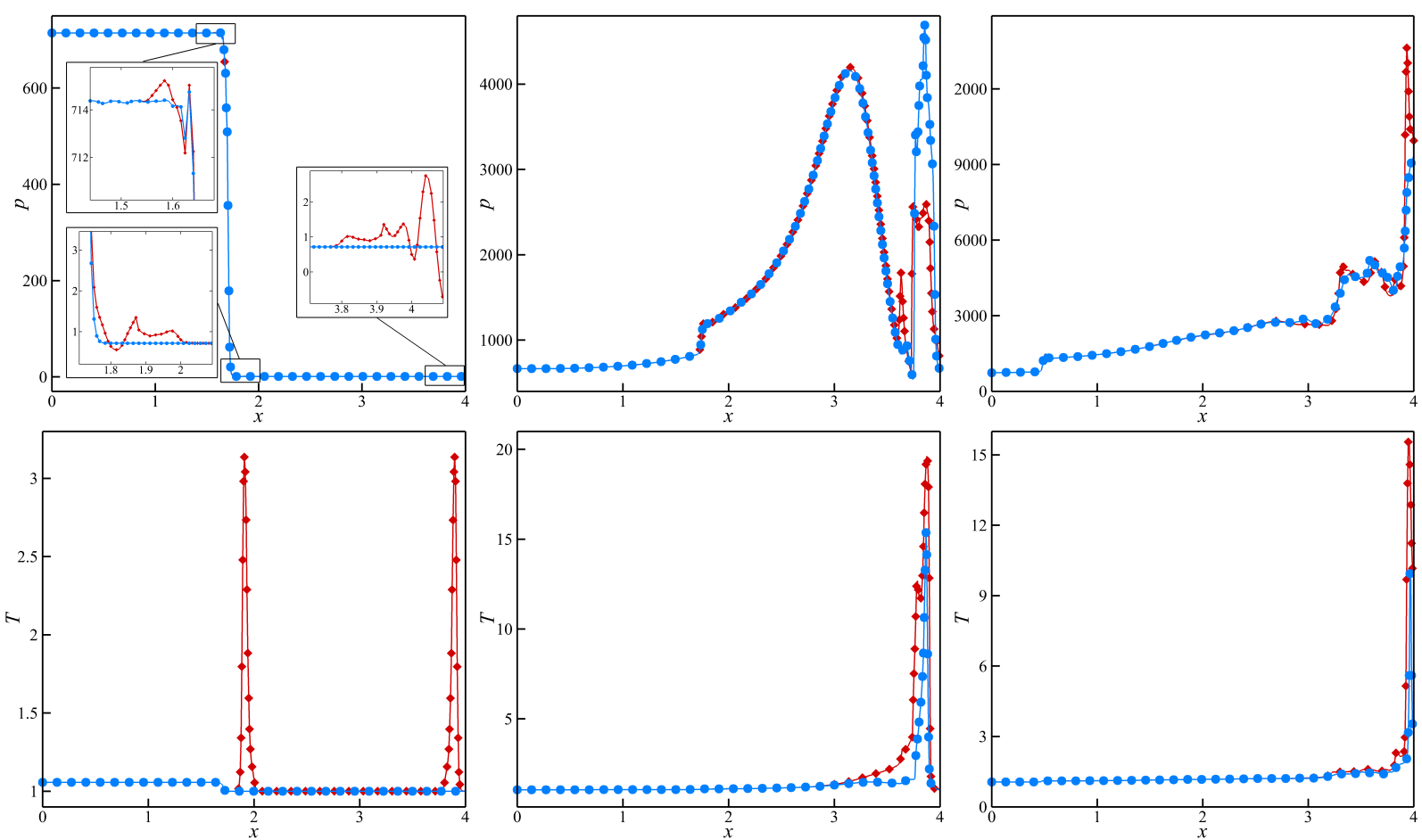

Figure 9: Shock-induced bubble collapse (case 2, $p_{s} / p_{o}=1000$ ) - Centerline properties (top row: pressure; bottom row: temperature) at times 0.03, 0.6, 0.73. Red diamonds: "pressure only" approach; blue circles: proposed approach.
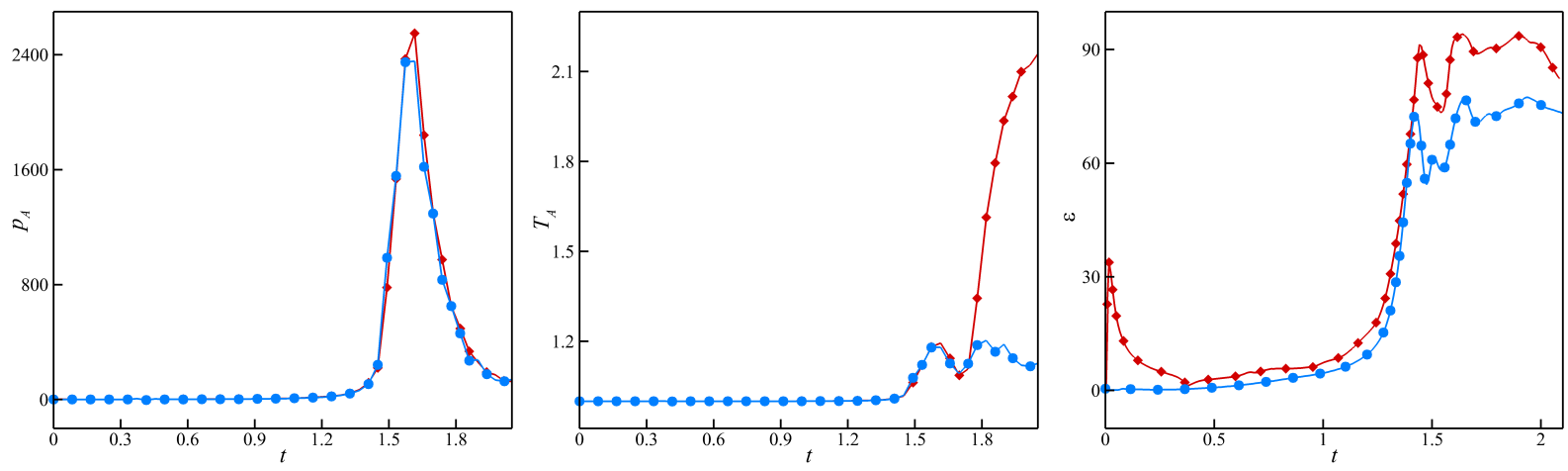

Figure 10: Shock-induced bubble collapse (case 1, $p_{s} / p_{o}=100$ ) - Time histories of the pressure (left) and temperature (center) at point $\mathrm{A}$ and total enstrophy (right). Red diamonds: "pressure only" approach; blue circles: proposed approach.

\section{Conclusions}

In this work, we identify and propose a strategy to prevent numerical errors produced by interfacecapturing schemes in simulations of gas/liquid interfaces with heat diffusion. We consider here the compressible Navier-Stokes equations and materials (gases, liquids and solids) that can be described by a stiffened equation of state. For the common $\gamma$, volume fraction and mass fraction models, we show that an incorrect 

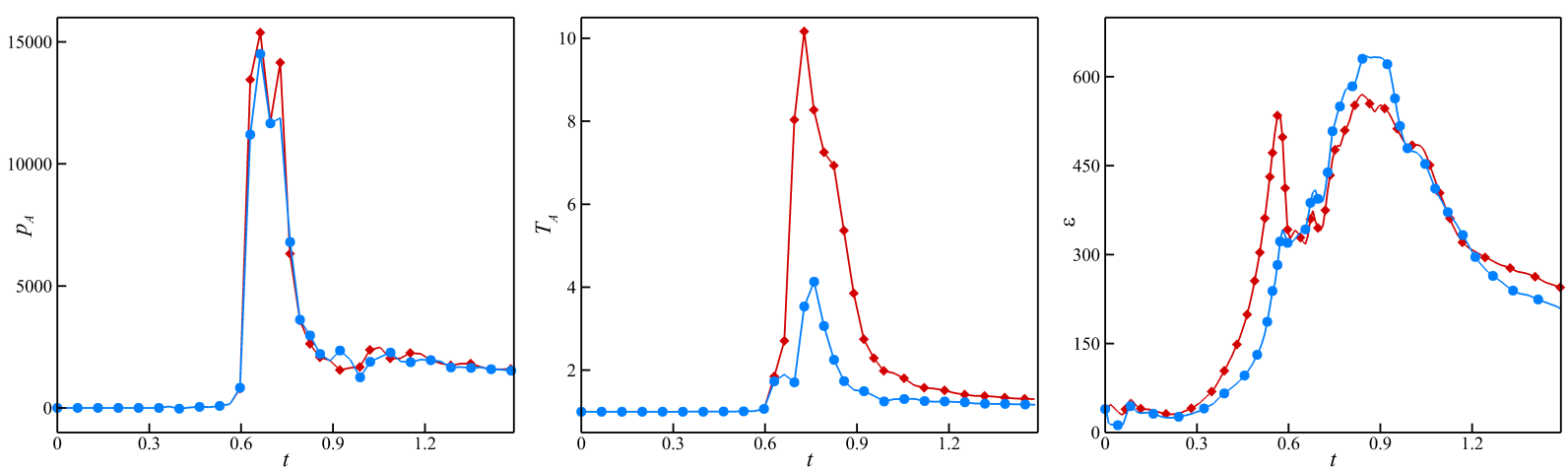

Figure 11: Shock-induced bubble collapse (case $\left.2, p_{s} / p_{o}=1000\right)$ - Time histories of the pressure (left) and temperature (center) at point A and total enstrophy (right). Red diamonds: "pressure only" approach; blue circles: proposed approach.

\section{Acknowledgements}

This research was supported by ONR grant N00014-12-1-0751 under Dr. Ki-Han Kim. 


\section{Appendix A.}

To derive the transport equations for the volume fraction formulation, we follow Miller \& Puckett [34] and start with Eq.(16), which can be expanded

$$
\frac{\partial \alpha^{(k)}}{\partial t}+\frac{\partial\left(\alpha^{(k)} u_{j}\right)}{\partial x_{j}}=-\frac{\alpha^{(k)}}{\rho^{(k)}} \frac{\partial \rho^{(k)}}{\partial t}-\frac{\alpha^{(k)} u_{j}}{\rho^{(k)}} \frac{\partial \rho^{(k)}}{\partial x_{j}} .
$$

We define the isentropic bulk modulus for each fluid, $K_{s}^{(k)}$, assuming isotropic stresses during advection and isentropic processes in any compression of the individual components. These assumptions imply that the pressure change associated with compression of the bulk $(\partial p)$ is equal to the pressure change associated with compression of each components $\left(\partial p^{(k)}\right)$. Then, $K_{s}^{(k)}$ can be defined as:

$$
K_{s}^{(k)}=\left.\frac{\partial p}{\partial \ln \rho^{(k)}}\right|_{s}=\left.\rho^{(k)} \frac{\partial p}{\partial \rho^{(k)}}\right|_{s}=\rho^{(k)}\left(a^{(k)}\right)^{2} .
$$

Differentiating Eq. (6) with respect to pressure and assuming constant entropies for each fluid results in:

$$
-\frac{1}{\rho}\left(\frac{1}{\left.\rho \frac{\partial p}{\partial \rho}\right|_{s}}\right)=-\sum_{k} \frac{z^{(k)}}{\rho^{(k)}}\left(\frac{1}{\left.\rho^{(k)} \frac{\partial p}{\partial \rho^{(k)}}\right|_{s}}\right) .
$$

By combining Eqs. (A.2) and (6), the isentropic bulk modulus and sound speed of the mixture are:

$$
\frac{1}{K_{s}}=\sum_{k} \frac{\alpha^{(k)}}{K_{s}^{(k)}}, \quad \frac{1}{\rho a^{2}}=\sum_{k} \frac{\alpha^{(k)}}{\rho^{(k)}\left(a^{(k)}\right)^{2}},
$$

thus recovering Wallis' relation [42]. According to the definition of isentropic bulk modulus and assuming pressure equilibrium between the phases, we can write $K_{s} \partial \rho / \rho=\partial p=\partial p^{(k)}=K_{s}^{(k)} \partial \rho^{(k)} / \partial \rho^{(k)}$. Using (16) 385 yields:

$$
\frac{\partial \alpha^{(1)}}{\partial t}+\frac{\partial\left(\alpha^{(1)} u_{j}\right)}{\partial x_{j}}=\frac{\alpha^{(1)} K_{s}}{K_{s}^{(1)}} \frac{\partial u_{j}}{\partial x_{j}}
$$

Finally, substituting Eqs. (A.2) and (A.4) into Eq. (A.5) yields, after appropriate manipulations:

$$
\frac{\partial \alpha^{(k)}}{\partial t}+u_{j} \frac{\partial \alpha^{(k)}}{\partial x_{j}}=\left(\frac{\alpha^{(k)} \alpha^{\left(k^{\prime}\right)}\left[\rho^{\left(k^{\prime}\right)}\left(a^{\left(k^{\prime}\right)}\right)^{2}-\rho^{(k)}\left(a^{(k)}\right)^{2}\right]}{\left[\alpha^{(k)} \rho^{\left(k^{\prime}\right)}\left(a^{\left(k^{\prime}\right)}\right)^{2}+\alpha^{\left(k^{\prime}\right)} \rho^{(k)}\left(a^{(k)}\right)^{2}\right]}\right) \frac{\partial u_{j}}{\partial x_{j}} .
$$

\section{References}

[1] J. Glimm, J. W. Grove, X. L. Li, K. M. Shyue, Y. Zeng, Q. Zhang, Three-Dimensional Front Tracking, SIAM Journal on Scientific Computing 19 (1998) 703-727. doi:10.1137/S1064827595293600.

[2] S. Osher, J. A. Sethian, Fronts propagating with curvature dependent speed: Algorithms based on Hamilton-Jacobi formulations, Journal of Computational Physics 49 (1987) 12-49. doi:10.1016/0021-9991(88)90002-2. 
[3] R. Abgrall, How to Prevent Pressure Oscillations in Multicomponent Flow Calculations: A Quasi Conservative Approach, Journal of Computational Physics 125 (1996) 150-160.

[12] E. Johnsen, T. Colonius, Numerical simulations of non-spherical bubble collapse, Journal of Fluid Mechanics 629 (2009) 231-261. doi:10.1017/S0022112009006351.

[13] V. Coralic, T. Colonius, Finite-volume WENO scheme for viscous compressible multicomponent flows, 420

[14] S. Kawai, H. Terashima, A high-resolution scheme for compressible multicomponent flows with shock waves, International Journal for Numerical Methods in Fluids 66 (March 2010) (2011) 1207-1225. doi:10.1002/fld.2306 
[15] H. Terashima, S. Kawai, M. Koshi, Consistent numerical diffusion terms for simulating compressible multicomponent flows, Computers and Fluids 88 (2013) 484-495. doi:10.1016/j.compfluid.2013.10.007

[16] E. Johnsen, F. Ham, Preventing numerical errors generated by interface-capturing schemes in compressible multi-material flows, Journal of Computational Physics 231 (2012) 5705-5717. doi:10.1016/j.jcp.2012.04.048.

[17] M. Baer, J. Nunziato, A two-phase mixture theory for the deflagration-to-detonation transition (ddt) in reactive granular materials, International Journal of Multiphase Flow 12 (1986) 861-889. doi:10.1016/0301-9322(86)90033-9

[18] A. K. Kapila, R. Menikoff, J. B. Bdzil, S. F. Son, D. S. Stewart, Two-phase modeling of deflagration-todetonation transition in granular materials: Reduced equations, Physics of Fluids 13 (2001) 3002-3024. doi:10.1063/1.1398042,

[19] G. Allaire, S. Clerc, S. Kokh, A Five-Equation Model for the Simulation of Interfaces between Compressible Fluids, Journal of Computational Physics 181 (2002) 577-616. doi:10.1006/jcph.2002.7143

[20] R. Saurel, R. Abgrall, A Multiphase Godunov Method for Compressible Multifluid and Multiphase Flows, Journal of Computational Physics 150 (1999) 425-467. doi:10.1006/jcph.1999.6187.

440 [21] G. Perigaud, R. Saurel, A compressible flow model with capillary effects, Journal of Computational Physics 209 (2005) 139-178. doi:10.1016/j.jcp.2005.03.018.

[22] J. J. Kreeft, B. Koren, A new formulation of Kapila's five-equation model for compressible twofluid flow, and its numerical treatment, Journal of Computational Physics 229 (2010) 6220-6242. doi:10.1016/j.jcp.2010.04.025.

445 [23] A. Murrone, H. Guillard, A five equation reduced model for compressible two phase flow problems, Journal of Computational Physics 202 (2005) 664-698. doi:10.1016/j.jcp.2004.07.019.

[24] F. Petitpas, J. Massoni, R. Saurel, E. Lapebie, L. Munier, Diffuse interface model for high speed cavitating underwater systems, International Journal of Multiphase Flow 35 (2009) 747-759. doi:10.1016/j.ijmultiphaseflow.2009.03.011.

[25] T. Flatten, A. Morin, S. T. Munkejord, Wave Propagation in Multicomponent Flow Models, SIAM Journal on Applied Mathematics 70 (2010) 2861-2882. doi:10.1137/090777700.

[26] B. Braconnier, B. Nkonga, An all-speed relaxation scheme for interface flows with surface tension, Journal of Computational Physics 228 (2009) 5722-5739. doi:10.1016/j.jcp.2009.04.046 
[27] G. H. Schnerr, I. H. Sezal, S. J. Schmidt, Numerical investigation of three-dimensional cloud cavitation with special emphasis on collapse induced shock dynamics, Physics of Fluids 20 (2008). doi:10.1063/1.2911039.

[28] K. H. Kim, G. Chahine, J. P. Franc, A. Karimi, Advanced Experimental and Numerical Techniques for Cavitation Erosion Prediction, Vol. 106, Springer, Netherlands, 2014. doi:10.1007/978-94-017-8539-6.

[29] O. Le Métayer, J. Massoni, R. Saurel, Modelling evaporation fronts with reactive Riemann solvers, Journal of Computational Physics 205 (2005) 567-610. doi:10.1016/j.jcp.2004.11.021.

[30] R. Saurel, F. Petitpas, R. Abgrall, Modelling phase transition in metastable liquids: application to cavitating and flashing flows, Journal of Fluid Mechanics 607 (2008) 313-350. doi:10.1017/S0022112008002061.

465 [31] E. Goncalvès, R. F. Patella, Numerical study of cavitating flows with thermodynamic effect, Computers and Fluids 39 (2010) 99-113. doi:10.1016/j.compfluid.2009.07.009

[32] K. M. Shyue, A Fluid-Mixture Type Algorithm for Compressible Multicomponent Flow with van der Waals Equation of State, Journal of Computational Physics 156 (1999) 43-88. doi:10.1006/jcph.2001.6801.

470 [33] R. K. Shukla, C. Pantano, J. B. Freund, An interface capturing method for the simulation of multi-phase compressible flows, Journal of Computational Physics 229 (2010) 7411-7439. doi:10.1016/j.jcp.2010.06.025.

[34] G. H. Miller, E. G. Puckett, A high-order Godunov method for multiple condensed phases, Journal of Computational Physics 128 (1996) 134-164. doi:10.1006/jcph.1996.0200

475 [35] R. Abgrall, V. Perrier, Asymptotic Expansion of a Multiscale Numerical Scheme for Compressible Multiphase Flow, Multiscale Modeling \& Simulation 5 (2006) 84-115. doi:10.1137/050623851.

[36] R. K. Shukla, Nonlinear preconditioning for efficient and accurate interface capturing in simulation of multicomponent compressible flows, Journal of Computational Physics 276 (2014) 508-540. doi:10.1016/j.jcp.2014.07.034.

480 [37] S. Gottlieb, C. W. Shu, Total variation diminishing Runge-Kutta schemes, Mathematics of Computation 67 (1996) 73-85. doi:10.1090/S0025-5718-98-00913-2.

[38] G. S. Jiang, C. W. Shu, Efficient implementation of weighted ENO schemes, Journal of Computational Physics 228 (1996) 202-228. doi:10.1006/jcph.1996.0130. 
[39] E. Johnsen, J. Larsson, A. V. Bhagatwala, W. H. Cabot, P. Moin, B. J. Olson, P. S. Rawat, S. K. Shankar, B. Sjögreen, H. C. Yee, X. Zhong, S. K. Lele, Assessment of high-resolution methods for numerical simulations of compressible turbulence with shock waves, Journal of Computational Physics 229 (2010) 1213-1237. doi:10.1016/j.jcp.2009.10.028

[40] A. Harten, P. D. Lax, B. Van Leer, On upstream differencing and Godunov-type schemes for hyperbolic conservation laws, SIAM Review 25 (1982) 35-61. doi:10.1137/1025002.

490 [41] R. Saurel, R. Abgrall, A Simple Method for Compressible Multifluid Flows, SIAM Journal on Scientific Computing 21 (1999) 1115-1145. doi:10.1137/S1064827597323749.

[42] G. B. Wallis, One-dimensional two-phase flow, New York, McGraw-Hill 1969. 